\title{
Geodesic Distances and Intrinsic Distances on Some Fractal Sets
}

\author{
Dedicated to Professor Ichiro Shigekawa on the occasion of his 60th birthday
}

\author{
by
}

Masanori Hino

\begin{abstract}
Given strong local Dirichlet forms and $\mathbb{R}^{N}$-valued functions on a metrizable space, we introduce the concepts of geodesic distance and intrinsic distance on the basis of these objects. They are defined in a geometric and an analytic way, respectively, and they are closely related to each other in some classical situations. In this paper, we study the relations of these distances when the underlying space has a fractal structure. In particular, we prove their coincidence for a class of self-similar fractals.
\end{abstract}

2010 Mathematics Subject Classification: Primary 31C25; Secondary 28A80, 31E05.

Keywords: geodesic distance, intrinsic distance, Dirichlet form, fractal, energy measure.

\section{§1. Introduction}

For the analysis of strong local Dirichlet forms $(\mathcal{E}, \mathcal{F})$ on a metric measure space $(K, \mu)$, the intrinsic distance defined as

$$
\mathrm{d}(x, y)=\sup \left\{f(y)-f(x) \mid f \in \mathcal{F}_{\mathrm{loc}} \cap C(K) \text { and } \mu_{\langle f\rangle} \leq \mu\right\}, \quad x, y \in K,
$$

often plays a crucial role. Here, $\mathcal{F}_{\text {loc }}$ represents the space of functions locally in $\mathcal{F}$ and $\mu_{\langle f\rangle}$ denotes the energy measure of $f$. For example, in a general framework, the off-diagonal Gaussian estimate and the Varadhan estimate of the transition density associated with $(\mathcal{E}, \mathcal{F})$ are described on the basis of the intrinsic distance (see, e.g., $[19,18,2]$ and the references therein). When the underlying space has a Riemannian structure, the geodesic distance $\rho(x, y)$ is also defined as the infimum

Communicated by T. Kumagai. Received November 7, 2012. Revised August 6, 2013, and October $11,2013$.

M. Hino: Graduate School of Engineering Science, Osaka University, Osaka 560-8531, Japan; e-mail: hino@sigmath.es.osaka-u.ac.jp 
of the lengths of continuous curves connecting $x$ and $y$, and $\mathrm{d}$ and $\rho$ coincide with each other under suitable conditions.

In this paper, we focus on the case when $K$ does not have a differential structure, in particular, when $K$ is a fractal set, and we study the relation between two distances that are defined in a way similar to $\mathrm{d}$ and $\rho$. The straightforward formulation of this problem, however, does not work well. This is because in typical examples such as the canonical Dirichlet forms on Sierpiński gaskets with the Hausdorff measure $\mu$, the energy measures are always singular to $\mu$ (see, e.g., [6, $10,15])$; accordingly, $\mathrm{d}$ vanishes everywhere. This is closely related to the fact that the transition density exhibits sub-Gaussian behavior. Nevertheless, if the reference measure in the definition of $d$ is replaced suitably, we can obtain a nontrivial intrinsic distance. Indeed, Kigami [14] and Kajino [11] studied, following Metz and Sturm [17], the canonical Dirichlet form on the 2-dimensional standard Sierpiński gasket with the underlying measure $\mu_{\left\langle h_{1}\right\rangle}+\mu_{\left\langle h_{2}\right\rangle}$, where the pair $h_{1}$ and $h_{2}$ is taken as the orthonormal system of the space of harmonic functions. In such a case, the mapping $\boldsymbol{h}:=\left(h_{1}, h_{2}\right): K \rightarrow \mathbb{R}^{2}$ provides a homeomorphism of $K$ onto its image ([12]). In particular, they proved that

- the intrinsic distance $\mathrm{d}_{\boldsymbol{h}}$ on $K$ coincides with the geodesic distance $\rho_{\boldsymbol{h}}$ on $\boldsymbol{h}(K)$ by identifying $K$ and $\boldsymbol{h}(K)$;

- the transition density associated with $(\mathcal{E}, \mathcal{F})$ on $L^{2}(K, \mu)$ has off-diagonal Gaussian estimates by using such distances.

In this paper, we study the relation between $d_{\boldsymbol{h}}$ and $\rho_{\boldsymbol{h}}$ (defined on $K$ ) in more general frameworks. First, we prove the one-sided inequality $\rho_{\boldsymbol{h}} \leq \mathrm{d}_{\boldsymbol{h}}$ when the underlying spaces have finitely ramified cell structures (Theorem 2.2). The reverse inequality is proved under tighter constraints on self-similar Dirichlet forms on a class of self-similar fractals (Theorem 2.3); typical examples are the standard Dirichlet forms on the 2-dimensional generalized Sierpiński gaskets. Both the proofs are based on purely analytic arguments, unlike the corresponding proof in [11], where detailed information on the transition density was utilized, together with probabilistic arguments. Our results are applicable to some examples in which the precise behaviors of the associated transition densities are not known.

The crucial part of the proof of Theorem 2.2 is that the truncated geodesic distance function based on $\boldsymbol{h}$ satisfies the conditions in the definition of $\mathrm{d}_{\boldsymbol{h}}$. To prove this claim, we show that a discrete version of the geodesic distance has some good estimates and that the limit function inherits them.

The proof of Theorem 2.3 is more tricky. The key lemma (Lemma 4.6) is an analog of the classical fact on a domain $D$ of $\mathbb{R}^{d}$, stating that any function $f \in W^{1,1}(D)$ with $|\nabla f|_{\mathbb{R}^{d}} \leq 1$ a.e. is locally Lipschitz with a local Lipschitz 
constant less than or equal to 1 . We prove that $\mathrm{d}_{\boldsymbol{h}}(x, y) \leq(1+\varepsilon) \rho_{\boldsymbol{h}}(x, y)$ if $x$ and $y$ are suitably located. An inequality of this type is not evident in the nonsmooth setting; the hidden obstacle is that a type of "Riemannian metric" which $K$ is equipped with is degenerate almost everywhere (cf. [8, 9, 15]), and we have $a$ priori the inequality stated above only for the points that are nondegenerate with respect to $\boldsymbol{h}$. Using a rather strong assumption ((B1) in Section 2), we can take sufficiently many such good points on arbitrary continuous curves, which enables us to deduce the inequality $\mathrm{d}_{\boldsymbol{h}} \leq \rho_{\boldsymbol{h}}$. At the moment, we need various assumptions to obtain such estimates owing to the lack of more effective tools for analysis. However, we expect the claims of the theorems in this paper to be valid in much more general situations, possibly with an appropriate modification of the framework (see also Remark 2.6 for further discussion).

The remainder of this article is organized as follows. In Section 2, we present the framework and state the main theorems. In Sections 3 and 4, we prove Theorems 2.2 , and 2.3 , respectively.

\section{§2. Framework and results}

Let $K$ be a compact metrizable space, and $\mu$, a finite Borel measure on $K$ with full support. Let $d_{K}$ denote a metric on $K$ that is compatible with the topology. For subsets $U$ of $K$, we denote the closure, interior, and boundary of $U$ by $\bar{U}$, $U^{\circ}$ and $\partial U$, respectively. The set of all real-valued continuous functions on $K$ is represented as $C(K)$, and is equipped with the uniform topology.

Let $(\mathcal{E}, \mathcal{F})$ be a strong local regular (symmetric) Dirichlet form on $L^{2}(K, \mu)$. For simplicity, we write $\mathcal{E}(f)$ for $\mathcal{E}(f, f)$. The space $\mathcal{F}$ is regarded as a Hilbert space with the inner product $(f, g)_{\mathcal{F}}:=\mathcal{E}(f, g)+\int_{K} f g d \mu$. For $f \in \mathcal{F}, \mu_{\langle f\rangle}$ denotes the energy measure of $f$, that is, when $f$ is bounded, $\mu_{\langle f\rangle}$ is characterized by the identity

$$
\int_{K} \varphi d \mu_{\langle f\rangle}=2 \mathcal{E}(f, f \varphi)-\mathcal{E}\left(f^{2}, \varphi\right) \text { for all } \varphi \in \mathcal{F} \cap C(K)
$$

for general $f \in \mathcal{F}, \mu_{\langle f\rangle}$ is defined by the natural approximation. Let $N \in \mathbb{N}$ and $\boldsymbol{h}=\left(h_{1}, \ldots, h_{N}\right)$ be such that $h_{j} \in \mathcal{F} \cap C(K)$ for every $j=1, \ldots, N$. Let $\mu_{\langle\boldsymbol{h}\rangle}$ denote $\sum_{j=1}^{N} \mu_{\left\langle h_{j}\right\rangle}$. Then the intrinsic distance based on $(\mathcal{E}, \mathcal{F})$ and $\mu_{\langle\boldsymbol{h}\rangle}$ is defined as

$$
\mathrm{d}_{\boldsymbol{h}}(x, y):=\sup \left\{f(y)-f(x) \mid f \in \mathcal{F} \cap C(K) \text { and } \mu_{\langle f\rangle} \leq \mu_{\langle\boldsymbol{h}\rangle}\right\}, \quad x, y \in K .
$$

We remark that the underlying measure $\mu$ does not play an essential role in (2.1). Further, we do not assume the absolute continuity of energy measures with respect 
to $\mu$ or $\mu_{\langle\boldsymbol{h}\rangle}$. For a continuous curve $\gamma \in C([s, t] \rightarrow K)$, its length based on $\boldsymbol{h}$ is defined as

$$
\ell_{\boldsymbol{h}}(\gamma):=\sup \left\{\sum_{i=1}^{n}\left|\boldsymbol{h}\left(\gamma\left(t_{i}\right)\right)-\boldsymbol{h}\left(\gamma\left(t_{i-1}\right)\right)\right|_{\mathbb{R}^{N}} \mid n \in \mathbb{N}, s=t_{0}<t_{1}<\cdots<t_{n}=t\right\},
$$

where $|\cdot|_{\mathbb{R}^{N}}$ denotes the Euclidean norm on $\mathbb{R}^{N}$. This is nothing but the pullback of the concept of the usual length of curves in $\mathbb{R}^{N}$ by the map $\boldsymbol{h}$. Then the geodesic distance based on $\boldsymbol{h}$ is defined as

$$
\rho_{\boldsymbol{h}}(x, y):=\inf \left\{\ell_{\boldsymbol{h}}(\gamma) \mid \gamma \in C([0,1] \rightarrow K), \gamma(0)=x, \text { and } \gamma(1)=y\right\}, \quad x, y \in K,
$$

where inf $\emptyset:=\infty$. If $\gamma \in C([s, t] \rightarrow K)$ satisfies $\gamma(s)=x, \gamma(t)=y$, and $\rho_{\boldsymbol{h}}(x, y)=$ $\ell_{\boldsymbol{h}}(\gamma)$, we say that $\gamma$ is a shortest path connecting $x$ and $y$.

We note that the two distances introduced here can be defined for more general underlying spaces such as locally compact spaces, by slight modifications if necessary. In this paper, however, we consider only compact spaces for simplicity.

Remark 2.1. We have the following properties.

(i) Both $\mathrm{d}_{\boldsymbol{h}}$ and $\rho_{\boldsymbol{h}}$ are $([0,+\infty]$-valued $)$ quasi-metrics on $K$, that is, the distance between two distinct points may be zero, but all the other axioms of metric are satisfied (see Corollary 3.14 for further discussion).

(ii) Let $\gamma \in C([s, t] \rightarrow K)$. If $\left\{s_{n}\right\}$ decreases to $s$ and $\left\{t_{n}\right\}$ increases to $t$, then $\lim _{n \rightarrow \infty} \ell_{\boldsymbol{h}}\left(\left.\gamma\right|_{\left[s_{n}, t_{n}\right]}\right)=\ell_{\boldsymbol{h}}(\gamma)$.

(iii) If $\gamma \in C([0,1] \rightarrow K)$ is a shortest path connecting $x$ and $y$ with $\rho_{\boldsymbol{h}}(x, y)<\infty$, then for any $0 \leq s<t \leq 1,\left.\gamma\right|_{[s, t]}$ is a shortest path connecting $\gamma(s)$ and $\gamma(t)$.

(iv) If $\boldsymbol{h}: K \rightarrow \mathbb{R}^{N}$ is injective, then for any $x, y \in K, \rho_{\boldsymbol{h}}(x, y)$ coincides with the geodesic distance between $\boldsymbol{h}(x)$ and $\boldsymbol{h}(y)$ in $\boldsymbol{h}(K) \subset \mathbb{R}^{N}$ on the basis of the Euclidean distance.

In order to state our first theorem, we consider the following conditions.

(A1) There exists an increasing sequence $\left\{V_{m}\right\}_{m=0}^{\infty}$ of nonempty finite subsets of $K$ such that:

(i) For each $m, K \backslash V_{m}$ is decomposed into finitely many connected components $\left\{U_{\lambda}\right\}_{\lambda \in \Lambda_{m}}$

(ii) For every $x \in K$, the sets $\left\{\bigcup_{\lambda \in \Lambda_{m}, x \in \overline{U_{\lambda}}} \overline{U_{\lambda}}\right\}_{m=0}^{\infty}$ constitute a fundamental system of neighborhoods of $x$.

(A2) $\mathcal{F} \subset C(K)$.

(A3) $\mathcal{E}(f, f)=0$ if and only if $f$ is a constant function. 
We give several remarks. In Lemma 3.3 below, it is proved from conditions (A1)(A3) that $K$ is arcwise connected. Then it is easy to prove that $V_{*}:=\bigcup_{m=0}^{\infty} V_{m}$ is dense in $K$. From the closed graph theorem, (A2) implies that $\mathcal{F}$ is continuously imbedded in $C(K)$. Condition (A3) is equivalent to the irreducibility of $(\mathcal{E}, \mathcal{F})$ in this framework, from [1, Theorem 2.1.11], for example. For $\lambda \in \Lambda_{m}$ and $\lambda^{\prime} \in \Lambda_{m^{\prime}}$ with $m \leq m^{\prime}$, either $U_{\lambda} \supset U_{\lambda^{\prime}}$ or $U_{\lambda} \cap U_{\lambda^{\prime}}=\emptyset$ holds. A slightly different version of (A1) was discussed in [21] and named finitely ramified cell structure.

The first main theorem is stated as follows.

Theorem 2.2. Suppose (A1)-(A3). Then $\rho_{\boldsymbol{h}}(x, y) \leq \mathrm{d}_{\boldsymbol{h}}(x, y)$ for all $x, y \in K$.

To obtain the reverse inequality, we need tighter constraints. Following Kigami [13], we introduce the concepts of post-critically finite self-similar sets and harmonic structures associated with them. Let $\mathbb{Z}_{+}$denote the set of all nonnegative integers. Let $S$ be a finite set with $\# S \geq 2$. For $i \in S$, let $\psi_{i}: K \rightarrow K$ be a continuous injective map. Set $\Sigma=S^{\mathbb{N}}$. For $i \in S$, we define a shift operator $\sigma_{i}: \Sigma \rightarrow \Sigma$ as $\sigma_{i}\left(\omega_{1} \omega_{2} \cdots\right)=i \omega_{1} \omega_{2} \cdots$. We assume that there exists a continuous surjective map $\pi: \Sigma \rightarrow K$ such that $\psi_{i} \circ \pi=\pi \circ \sigma_{i}$ for every $i \in S$. Then $\mathcal{L}=\left(K, S,\left\{\psi_{i}\right\}_{i \in S}\right)$ is called a self-similar structure.

We set $W_{0}=\{\emptyset\}$ and $W_{m}=S^{m}$ for $m \in \mathbb{N}$. For $w=w_{1} \cdots w_{m} \in W_{m}$, let $\psi_{w}$ denote $\psi_{w_{1}} \circ \cdots \circ \psi_{w_{m}}$ and let $K_{w}$ denote $\psi_{w}(K)$. By convention, $\psi_{\emptyset}$ is the identity map from $K$ to $K$. Let

$$
\mathcal{P}=\bigcup_{m=1}^{\infty} \sigma^{m}\left(\pi^{-1}\left(\bigcup_{i, j \in S, i \neq j}\left(K_{i} \cap K_{j}\right)\right)\right) \quad \text { and } \quad V_{0}=\pi(\mathcal{P})
$$

where $\sigma^{m}: \Sigma \rightarrow \Sigma$ is defined as $\sigma^{m}\left(\omega_{1} \omega_{2} \cdots\right)=\omega_{m+1} \omega_{m+2} \cdots$. The set $\mathcal{P}$ is called the post-critical set. We assume that $K$ is connected and that the selfsimilar structure $\mathcal{L}$ is post-critically finite (p.c.f.), that is, $\mathcal{P}$ is a finite set. For $m \in \mathbb{N}$, let $V_{m}=\bigcup_{w \in W_{m}} \psi_{w}\left(V_{0}\right)$.

For a finite set $V, l(V)$ denotes the space of all real-valued functions on $V$. We equip $l(V)$ with an inner product $(\cdot, \cdot)_{l(V)}$ defined by $(u, v)_{l(V)}=\sum_{q \in V} u(q) v(q)$. The norm derived from $(\cdot, \cdot)_{l(V)}$ is denoted by $|\cdot|_{l(V)}$. Let $D=\left(D_{q q^{\prime}}\right)_{q, q^{\prime} \in V_{0}}$ be a symmetric linear operator on $l\left(V_{0}\right)$ (also considered to be a square matrix of size $\left.\# V_{0}\right)$ such that the following conditions hold:

(D1) $D$ is nonpositive definite.

(D2) $D u=0$ if and only if $u$ is constant on $V_{0}$.

(D3) $D_{q q^{\prime}} \geq 0$ for all $q \neq q^{\prime} \in V_{0}$.

We define $\mathcal{E}^{(0)}(u, v)=(-D u, v)_{l\left(V_{0}\right)}$ for $u, v \in l\left(V_{0}\right)$. This is a Dirichlet form on the $L^{2}$ space on $V_{0}$ with the counting measure (cf. [13, Proposition 2.1.3]). For 
$\boldsymbol{r}=\left\{r_{i}\right\}_{i \in S}$ with $r_{i}>0$, we define a bilinear form $\mathcal{E}^{(m)}$ on $l\left(V_{m}\right)$ as

$$
\mathcal{E}^{(m)}(u, v)=\sum_{w \in W_{m}} \frac{1}{r_{w}} \mathcal{E}^{(0)}\left(\left.u \circ \psi_{w}\right|_{V_{0}},\left.v \circ \psi_{w}\right|_{V_{0}}\right), \quad u, v \in l\left(V_{m}\right) .
$$

Here, $r_{w}=r_{w_{1}} \cdots r_{w_{m}}$ for $w=w_{1} \cdots w_{m} \in W_{m}$ and $r_{\emptyset}=1$. We call $(D, \boldsymbol{r})$ a regular harmonic structure if $0<r_{i}<1$ for all $i \in S$ and

$$
\mathcal{E}^{(0)}(v, v)=\inf \left\{\mathcal{E}^{(1)}(u, u) \mid u \in l\left(V_{1}\right) \text { and }\left.u\right|_{V_{0}}=v\right\}
$$

for every $v \in l\left(V_{0}\right)$. Then $\mathcal{E}^{(m)}\left(\left.u\right|_{V_{m}},\left.u\right|_{V_{m}}\right) \leq \mathcal{E}^{(m+1)}(u, u)$ for $m \in \mathbb{Z}_{+}$and $u \in l\left(V_{m+1}\right)$. The existence of harmonic structures is a nontrivial problem. It is known that all nested fractals have canonical regular harmonic structures ([16]; see also [13]).

We assume that a regular harmonic structure $(D, \boldsymbol{r})$ is given. Let $\mu$ be a finite Borel measure on $K$ with full support. We can then define a strong local and regular Dirichlet form $(\mathcal{E}, \mathcal{F})$ on $L^{2}(K, \mu)$ associated with $(D, \boldsymbol{r})$ by

$$
\begin{aligned}
\mathcal{F} & =\left\{u \in C(K) \subset L^{2}(K, \mu) \mid \lim _{m \rightarrow \infty} \mathcal{E}^{(m)}\left(\left.u\right|_{V_{m}},\left.u\right|_{V_{m}}\right)<\infty\right\}, \\
\mathcal{E}(u, v) & =\lim _{m \rightarrow \infty} \mathcal{E}^{(m)}\left(\left.u\right|_{V_{m}},\left.v\right|_{V_{m}}\right), \quad u, v \in \mathcal{F}
\end{aligned}
$$

(see the beginning of [13, Section 3.4]). Then conditions (A1)-(A3) are satisfied. ((A1) is guaranteed by [13, Propositions 1.6.8(2) and 1.3.6].)

For a map $\psi: K \rightarrow K$ and a function $f$ on $K, \psi^{*} f$ denotes the pullback of $f$ by $\psi$, that is, $\psi^{*} f=f \circ \psi$. The Dirichlet form $(\mathcal{E}, \mathcal{F})$ constructed above satisfies the self-similarity

$$
\mathcal{E}(f, g)=\sum_{i \in S} \frac{1}{r_{i}} \mathcal{E}\left(\psi_{i}^{*} f, \psi_{i}^{*} g\right), \quad f, g \in \mathcal{F}
$$

For each $u \in l\left(V_{0}\right)$, there exists a unique function $h \in \mathcal{F}$ such that $\left.h\right|_{V_{0}}=u$ and $\mathcal{E}(h)=\inf \left\{\mathcal{E}(g)|g \in \mathcal{F}, g|_{V_{0}}=u\right\}$. Such a function $h$ is termed a harmonic function. The space of all harmonic functions is denoted by $\mathcal{H}$. For any $w \in W_{*}$ and $h \in \mathcal{H}$, we have $\psi_{w}^{*} h \in \mathcal{H}$. We can identify $\mathcal{H}$ with $l\left(V_{0}\right)$ by the linear map $\iota: l\left(V_{0}\right) \ni u \mapsto h \in \mathcal{H}$. In particular, $\mathcal{H}$ is a finite-dimensional subspace of $\mathcal{F}$. For each $i \in S$, we define a linear operator $A_{i}: l\left(V_{0}\right) \rightarrow l\left(V_{0}\right)$ as $A_{i}=\iota^{-1} \circ \psi_{i}^{*} \circ \iota$, which is also considered as a square matrix of size $\# V_{0}$. For $i \neq j \in S$, the fixed points $p_{i}$ and $p_{j}$ of $\psi_{i}$ and $\psi_{j}$, respectively, are different by [13, Lemma 1.3.14]. We set

$$
S_{0}=\left\{i \in S \mid \text { the fixed point } p_{i} \text { of } \psi_{i} \text { belongs to } V_{0}\right\} \text {. }
$$


For $i \in S_{0}, r_{i}$ is an eigenvalue of $A_{i}$, and we can take its eigenvector $v_{i} \in l\left(V_{0}\right)$ whose components are all nonnegative (cf. [13, Theorem A.1.2]). Note that we have $v_{i}\left(p_{i}\right)=0$ since $r_{i} \neq 1$.

We now consider the following conditions:

(B1) $\# V_{0}=3$.

(B2) For all $p \in V_{0}, K \backslash\{p\}$ is connected.

(B3) $\# S_{0}=3$, that is, each $p \in V_{0}$ is a fixed point of $\psi_{i}$ for some $i \in S_{0}$. Moreover, $D v_{i}(q)<0$ for every $q \in V_{0} \backslash\{p\}$.

(B4) For every $i \in S_{0}, A_{i}$ is invertible.

We remark that, in (B3), $v_{i}(q)>0$ follows in addition for every $q \in V_{0} \backslash\{p\}$ from (B2) and [13, Corollary A.1.3].

Theorem 2.3. Suppose (B1)-(B4). Take $\boldsymbol{h}=\left(h_{1}, \ldots, h_{N}\right)$ such that each $h_{j}$ is a harmonic function. Then $\mathrm{d}_{\boldsymbol{h}}(x, y)=\rho_{\boldsymbol{h}}(x, y)$ for all $x, y \in K$.

Typical examples that meet conditions (B1)-(B4) are given below.

Example 2.4. Take the 2-dimensional level $l$ Sierpiński gasket as $K$ (see Figure 1). The set $V_{0}$ consists of the three vertices $p_{1}, p_{2}$, and $p_{3}$ of the largest triangle in $K$. For $i=1,2,3$, let $\psi_{i}$ denote the map whose fixed point is $p_{i}$ among the contraction maps constructing $K$. Since $K$ is a nested fractal, there exists a canonical regular harmonic structure $(D, \boldsymbol{r})$ corresponding to the Brownian motion on $K$. The matrix $D$ is given by

$$
D=\left(D_{p_{i} p_{j}}\right)_{i, j=1}^{3}=\left[\begin{array}{ccc}
-2 & 1 & 1 \\
1 & -2 & 1 \\
1 & 1 & -2
\end{array}\right] .
$$

By symmetry the eigenvector $v_{1}$ is

$$
v_{1}=\left[\begin{array}{l}
0 \\
1 \\
1
\end{array}\right] ; \quad \text { thus, } D v_{1}=\left[\begin{array}{c}
2 \\
-1 \\
-1
\end{array}\right] .
$$

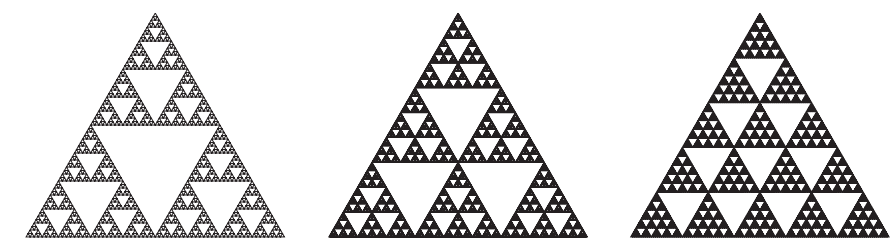

Figure 1. 2-dimensional level $l$ Sierpiński gaskets $(l=2,3,5)$. 
Similarly, the vectors $v_{i}$ and $D v_{i}$ for $i=2,3$ are

$$
v_{2}=\left[\begin{array}{l}
1 \\
0 \\
1
\end{array}\right], \quad D v_{2}=\left[\begin{array}{c}
-1 \\
2 \\
-1
\end{array}\right], \quad v_{3}=\left[\begin{array}{l}
1 \\
1 \\
0
\end{array}\right], \quad D v_{3}=\left[\begin{array}{c}
-1 \\
-1 \\
2
\end{array}\right] \text {. }
$$

Therefore, conditions (B1)-(B3) hold. Condition (B4) is also verified directly. We note that the detailed information on the transition density associated with $(\mathcal{E}, \mathcal{F})$ on $L^{2}\left(K, \mu_{\langle\boldsymbol{h}\rangle}\right)$ is known only for $l=2$ (see $[14,11]$ ), since we cannot expect the volume doubling property of $\mu_{\langle\boldsymbol{h}\rangle}$ if $l \geq 3$.

The following examples are based on the suggestion of the referee.

Example 2.5. Let $n$ be 6 or 9 . Let $S=\{0,1, \ldots, n-1\}$ and $p_{k}=\exp (2 \pi k \sqrt{-1} / n)$ for $k \in \mathbb{Z}$. For $k \in S$, we define $\psi_{k}: \mathbb{C} \rightarrow \mathbb{C}$ by

$$
\psi_{k}(z)=p_{k}\left\{\beta_{n}(z-1)+1\right\}
$$

where $\beta_{n}=2 /(3+\sqrt{3} \cot (\pi / n))$. Let $K$ be the unique nonempty compact subset of $\mathbb{C}$ such that $K=\bigcup_{k \in S} \psi_{k}(K)$ (see Figure 2 and also [20, Example 7.4]). Then the triplet $\left(K, S,\left\{\left.\psi_{k}\right|_{K}\right\}_{k \in S}\right)$ constitutes a self-similar structure, $\# \mathcal{P}=3$, and $V_{0}=\left\{p_{0}, p_{n / 3}, p_{2 n / 3}\right\}$. Note that $\beta_{n}$ is taken so that $\psi_{0}(K) \cap \psi_{1}(K)$ is a one-point set, and that $\# V_{0}$ is not $n$ but 3 since $\psi_{j}$ involves a rotation. We can construct a canonical harmonic structure as in Example 2.4 such that conditions (B1)-(B4) hold.
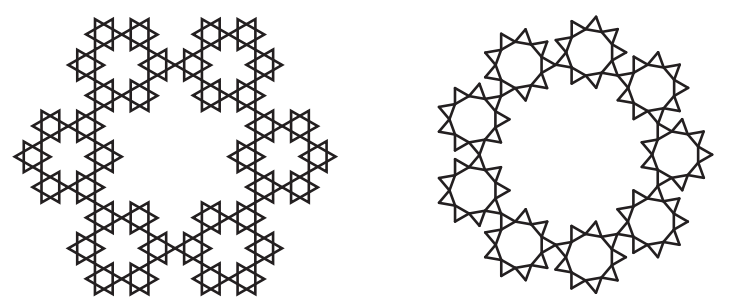

Figure 2. Hexagasket $(n=6)$ and Nonagasket $(n=9)$.

Remark 2.6. Let us consider the classical case for comparison. Let $K$ be a nonempty compact set of $\mathbb{R}^{m}$ such that $\overline{K^{\circ}}=K$ and $\partial K$ is a smooth hypersurface. Let $(\mathcal{E}, \mathcal{F})$ be the Dirichlet form on $L^{2}(K, d x)$ given by

$$
\mathcal{E}(f, g)=\frac{1}{2} \int_{K} \sum_{i, j=1}^{m} a_{i j}(x) \frac{\partial f}{\partial x_{i}}(x) \frac{\partial g}{\partial x_{j}}(x) d x, \quad f, g \in \mathcal{F}:=H^{1}(K) .
$$

Here, $A(x)=\left(a_{i j}(x)\right)_{i, j=1}^{m}$ is a symmetric, bounded, and uniformly positive definite matrix-valued continuous function on $K$. Let $N \geq m$ and let $h_{i}$ be a Lipschitz 
function on $K$ for $i=1, \ldots, N$. Let $B$ be an $N \times m$-matrix valued function on $K$ such that the $i$ th row of $B(x)$ is equal to ${ }^{t} \nabla h_{i}(x)$ for $i=1, \ldots, N$ and $x \in K$. Assume that $A(x)$ is connected $\left(h_{1}, \ldots, h_{N}\right)$ by the identity ${ }^{t} B(x) B(x)=A(x)^{-1}$ for a.e. $x$. Then

$$
\begin{aligned}
d \mu_{\langle\boldsymbol{h}\rangle} & =\sum_{i=1}^{N}\left(A(x) \nabla h_{i}(x), \nabla h_{i}(x)\right)_{\mathbb{R}^{m}} d x=\sum_{i=1}^{N}{ }^{t} \nabla h_{i}(x) A(x) \nabla h_{i}(x) d x \\
& =\operatorname{tr}\left(B(x) A(x)^{t} B(x)\right) d x=\operatorname{tr}\left(A(x){ }^{t} B(x) B(x)\right) d x=m \cdot d x .
\end{aligned}
$$

Therefore, $\mathrm{d}_{\boldsymbol{h}}(x, y)$ should be defined as

$$
\begin{aligned}
& \mathrm{d}_{\boldsymbol{h}}(x, y) \\
& =\sup \left\{f(y)-f(x) \mid f \in H^{1}(K) \cap C(K) \text { and }(A(z) \nabla f(z), \nabla f(z))_{\mathbb{R}^{m}} \leq m \text { a.e. } z\right\} .
\end{aligned}
$$

Moreover, for $\boldsymbol{b} \in \mathbb{R}^{m}$ and $x \in K$,

$$
\sum_{i=1}^{N}\left(\nabla h_{i}(x), \boldsymbol{b}\right)_{\mathbb{R}^{m}}^{2}=|B(x) \boldsymbol{b}|_{\mathbb{R}^{N}}^{2}={ }^{t} \boldsymbol{b}^{t} B(x) B(x) \boldsymbol{b}=\left|A(x)^{-1 / 2} \boldsymbol{b}\right|_{\mathbb{R}^{m}}^{2},
$$

which implies that for $\gamma \in C([0,1] \rightarrow K)$ that is piecewise smooth,

$$
\begin{aligned}
\ell_{\boldsymbol{h}}(\gamma) & =\int_{0}^{1}\left|\frac{d}{d t}(\boldsymbol{h} \circ \gamma)(t)\right|_{\mathbb{R}^{N}} d t=\int_{0}^{1}\left(\sum_{i=1}^{N}\left(\left(\nabla h_{i}\right)(\gamma(t)), \dot{\gamma}(t)\right)_{\mathbb{R}^{m}}^{2}\right)^{1 / 2} d t \\
& =\int_{0}^{1}\left|A(\gamma(t))^{-1 / 2} \dot{\gamma}(t)\right|_{\mathbb{R}^{m}} d t .
\end{aligned}
$$

Thus, $\mathrm{d}_{\boldsymbol{h}}(x, y)=\sqrt{m} \rho_{\boldsymbol{h}}(x, y) .{ }^{1}$ This example shows that the information on the dimension of $K$ is required to identify $\mathrm{d}_{\boldsymbol{h}}$ with $\rho_{\boldsymbol{h}}$ in general. The author guesses that the correct measure to define the intrinsic metric is $p(x)^{-1} d \mu_{\langle\boldsymbol{h}\rangle}$ instead

\footnotetext{
${ }^{1}$ Though this type of identity ought to be a known result (cf. [3]), we give a sketch of the proof. For $x \in K$ and $M>0$, the function $f:=\rho_{\boldsymbol{h}}(\cdot, y) \wedge M$ on $K$ is locally Lipschitz and $\left|(\nabla f(x), \boldsymbol{b})_{\mathbb{R}^{m}}\right| \leq\left|A(x)^{-1 / 2} \boldsymbol{b}\right|_{\mathbb{R}^{m}}$ for any $\boldsymbol{b} \in \mathbb{R}^{m}$. By letting $\boldsymbol{b}=A(x) \nabla f(x)$, we obtain $\left|A(x)^{1 / 2} \nabla f(x)\right|_{\mathbb{R}^{m}} \leq 1$. This implies that $\rho_{\boldsymbol{h}}(x, y) \wedge M \leq \mathrm{d}_{\boldsymbol{h}}(x, y) / \sqrt{m}$ for $x \in K$. On the other hand, for $f \in C^{1}(K)$,

$$
f(y)-f(x)=\int_{0}^{1}(\nabla f(\gamma(t)), \dot{\gamma}(t))_{\mathbb{R}^{m}} d t \leq \int_{0}^{1}\left|A(\gamma(t))^{1 / 2} \nabla f(\gamma(t))\right|_{\mathbb{R}^{m}}\left|A(\gamma(t))^{-1 / 2} \dot{\gamma}(t)\right|_{\mathbb{R}^{m}} d t .
$$

Let $\gamma \in C([0,1] \rightarrow K)$ be a piecewise-linear curve connecting $x$ and $y$, and $f$ a function on $K$ satisfying the condition in the definition of $d_{h}$. Then

$$
f(y)-f(x) \leq \sqrt{m} \int_{0}^{1}\left|A(\gamma(t))^{-1 / 2} \dot{\gamma}(t)\right|_{\mathbb{R}^{m}} d t=\sqrt{m} \ell_{\boldsymbol{h}}(\gamma)
$$

(approximate $f$ by smooth functions if necessary). Taking the supremum and infimum with respect to $f$ and $\gamma$, respectively, we conclude that $\mathrm{d}_{\boldsymbol{h}}(x, y) \leq \sqrt{m} \rho_{\boldsymbol{h}}(x, y)$.
} 
of $\mu_{\langle\boldsymbol{h}\rangle}$, where $p(x)$ is the pointwise index defined in [8] and represents the effective dimension of a type of tangent space at $x$ (see also [9]); the treatment of such a measure is beyond the scope of this paper. Under the assumptions of Theorem 2.3, $p(x)=1$ for $\mu_{\langle\boldsymbol{h}\rangle}$-a.e. $x$ from the result of [7]. Therefore, this guess is also consistent with Theorem 2.3. Such examples show that the problem discussed in this paper is more intricate than it seems.

\section{§3. Proof of Theorem 2.2}

First, we record some properties of energy measures associated with strong local Dirichlet forms. For the proof, see [1, Theorem 4.3.8], for example. In the statement of Lemma 3.1, $\tilde{f}$ denotes the quasi-continuous modification of $f$.

Lemma 3.1. For every $f \in \mathcal{F}$, the push-forward measure of $\mu_{\langle f\rangle}$ by $\tilde{f}$ is absolutely continuous with respect to the 1-dimensional Lebesgue measure. In particular, we have the following:

(i) For any $f \in \mathcal{F}, \mu_{\langle f\rangle}$ does not have a point mass.

(ii) For $f, g \in \mathcal{F}, \mu_{\langle f\rangle}=\mu_{\langle g\rangle}$ on the set $\{\tilde{f}=\tilde{g}\}$.

In this paper, we consider only the case $\mathcal{F} \subset C(K)$; accordingly, any $f \in \mathcal{F}$ is continuous from the beginning.

We also remark that

$$
\mathcal{E}(f)=\frac{1}{2} \mu_{\langle f\rangle}(K) \quad \text { for any } f \in \mathcal{F}
$$

(see [5, Corollary 3.2.1]).

For $f, g$ in $\mathcal{F}$, a signed measure $\mu_{\langle f, g\rangle}$ on $K$ is defined as $\mu_{\langle f, g\rangle}=$ $\left(\mu_{\langle f+g\rangle}-\mu_{\langle f\rangle}-\mu_{\langle g\rangle}\right) / 2$. It is bilinear in $f, g$, and we have $\mu_{\langle f, f\rangle}=\mu_{\langle f\rangle}$.

In the remainder of this section, we always assume (A1)-(A3). We state some basic properties in the following series of lemmas.

Lemma 3.2. Let $m \in \mathbb{Z}_{+}$and $\lambda \in \Lambda_{m}$. Then $U_{\lambda}$ is open, $\overline{U_{\lambda}} \subset U_{\lambda} \cup V_{m}$, and $\partial U_{\lambda} \subset V_{m}$.

Proof. If $\overline{U_{\lambda}} \cap U_{\kappa} \neq \emptyset$ for some $\kappa \in \Lambda_{m} \backslash\{\lambda\}$, then $U_{\lambda} \cup U_{\kappa}$ is connected (see, e.g., [4, Theorem 6.1.9]), which is a contradiction. Thus, $\overline{U_{\lambda}} \subset K \backslash \bigcup_{\kappa \in \Lambda_{m} \backslash\{\lambda\}} U_{\kappa}=$ $U_{\lambda} \cup V_{m}$. Similarly, we have $K \backslash U_{\lambda}=V_{m} \cup \bigcup_{\kappa \in \Lambda_{m} \backslash\{\lambda\}} \overline{U_{\kappa}}$, which is a closed set. Since $\partial U_{\lambda}=\overline{U_{\lambda}} \backslash U_{\lambda} \subset V_{m}$, the last claim follows.

Lemma 3.3. $K$ is arcwise connected. 
Proof. First, we prove that $K$ is connected. If $K$ is a disjoint union of nonempty open sets $K_{1}$ and $K_{2}$, then $\mathbf{1}_{K_{1}} \in \mathcal{F}$ from the regularity of $(\mathcal{E}, \mathcal{F})$. From the strong locality of $(\mathcal{E}, \mathcal{F}), \mathcal{E}\left(\mathbf{1}_{K_{1}}\right)=0$, which contradicts (A3).

Thus, $K$ is a compact, metrizable, connected, and locally connected space, which implies that $K$ is arcwise connected (see, e.g., [4, Section 6.3.11]).

For a subset $U$ of $K$, let $\operatorname{diam} U$ denote the diameter of $U$ with respect to the metric $d_{K}$.

Lemma 3.4. As $m \rightarrow \infty, \sup \left\{\operatorname{diam} \overline{U_{\lambda}} \mid \lambda \in \Lambda_{m}\right\}$ converges to 0 .

Proof. Let $\varepsilon>0$. From (A1)(ii), for each $x \in K$, there exists $m(x) \in \mathbb{Z}_{+}$such that

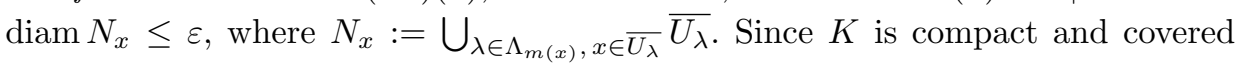
with $\bigcup_{x \in K} N_{x}^{\circ}, K$ can be written as $\bigcup_{j=1}^{k} N_{x_{j}}^{\circ}$ for some $\left\{x_{j}\right\}_{j=1}^{k} \subset K$. Let $M=$ $\max \left\{m\left(x_{j}\right) \mid j=1, \ldots, k\right\}$. Then, for each $m \geq M$ and $\lambda \in \Lambda_{m}, U_{\lambda}$ is a subset of some $N_{x_{j}}$, which implies that $\operatorname{diam} U_{\lambda} \leq \varepsilon$. This proves the claim.

Lemma 3.5. For a continuous curve $\gamma \in C([s, t] \rightarrow K)$,

$\ell_{\boldsymbol{h}}(\gamma)=\sup \left\{\begin{array}{l|l}\sum_{i=1}^{n}\left|\boldsymbol{h}\left(\gamma\left(t_{i}\right)\right)-\boldsymbol{h}\left(\gamma\left(t_{i-1}\right)\right)\right|_{\mathbb{R}^{N}} & \begin{array}{l}n \in \mathbb{N}, s=t_{0}<t_{1}<\cdots<t_{n}=t, \\ \gamma\left(t_{i}\right) \in V_{*} \text { for every } i=1, \ldots, n-1\end{array}\end{array}\right\}$.

Proof. This is evident from the fact that the set $\left\{u \in[s, t] \mid \gamma(u) \in V_{*}\right\}$ is dense in $[s, t]$ if $\gamma$ is not constant on any nonempty interval.

Lemma 3.6. Let $V$ be a finite and nonempty subset of $K$ and $u \in l(V)$. Then there exists a unique function $g \in \mathcal{F}$ such that $g$ attains the infimum of the set $\{\mathcal{E}(f) \mid f \in \mathcal{F}, f=u$ on $V\}$.

Proof. The proof is standard. From the regularity of $(\mathcal{E}, \mathcal{F})$, for each $p \in V$, there exists $g \in \mathcal{F}$ such that $g(p)=1$ and $g(q)=0$ for all $q \in V \backslash\{p\}$. Therefore, the set in the statement is nonempty. Take functions $\left\{f_{n}\right\}$ from $\mathcal{F}$ such that $f_{n}=u$ on $V, \min _{x \in V} u(x) \leq f_{n} \leq \max _{x \in V} u(x)$, and $\mathcal{E}\left(f_{n}\right)$ converges to $\inf \{\mathcal{E}(f) \mid f \in \mathcal{F}$, $f=u$ on $V\}$. Since $\left\{f_{n}\right\}$ is bounded in $\mathcal{F}$, we can take a subsequence of $\left\{f_{n}\right\}$ whose Cesàro means converge to some $g$ in $\mathcal{F}$. Then $g$ attains the infimum. If another $g^{\prime} \in \mathcal{F}$ attains the infimum, then $\mathcal{E}\left(g-g^{\prime}\right)=2 \mathcal{E}(g)+2 \mathcal{E}\left(g^{\prime}\right)-4 \mathcal{E}\left(\left(g+g^{\prime}\right) / 2\right) \leq 0$. From (A3), $g-g^{\prime}$ is a constant function. Since $g-g^{\prime}=0$ on $V$, we conclude that $g=g^{\prime}$, which ensures uniqueness of the minimizer.

For $m \in \mathbb{Z}_{+}$and $u \in l\left(V_{m}\right)$, let $H_{m} u$ denote the function $g$ in the above lemma with $V=V_{m}$. For $m \in \mathbb{Z}_{+}$and $f \in \mathcal{F}$, let $H_{m} f$ denote $H_{m}\left(\left.f\right|_{V_{m}}\right)$ by abuse of notation. The linearity of $H_{m}$ is a basic fact and its proof is omitted. 
Lemma 3.7. Let $U$ be an open set of $K$, and let $f$ be a function in $\mathcal{F}$ such that $f=0$ on $\partial U$. Then the function $f \cdot \mathbf{1}_{U}$ belongs to $\mathcal{F}$.

Proof. We may assume that $f$ is nonnegative. For $\varepsilon>0$, let $f_{\varepsilon}=(f-\varepsilon) \vee 0$. Then $f_{\varepsilon}=0$ on a certain open neighborhood $O_{\varepsilon}$ of $\partial U$. Take $\varphi_{\varepsilon} \in \mathcal{F}$ such that $\varphi_{\varepsilon}=1$ on $U \backslash O_{\varepsilon}$ and $\varphi_{\varepsilon}=0$ on $K \backslash \bar{U}$. Then $f_{\varepsilon} \cdot \mathbf{1}_{U}=f_{\varepsilon} \varphi_{\varepsilon} \in \mathcal{F}$. From Lemma 3.1 and $(3.1)$,

$$
\mathcal{E}\left(f_{\varepsilon} \cdot \mathbf{1}_{U}\right)=\frac{1}{2} \mu_{\left\langle f_{\varepsilon} \cdot \mathbf{1}_{U}\right\rangle}(U)=\frac{1}{2} \mu_{\left\langle f_{\varepsilon}\right\rangle}(U) \leq \mathcal{E}\left(f_{\varepsilon}\right) \leq \mathcal{E}(f) .
$$

Therefore, $\left\{f_{1 / n} \cdot \mathbf{1}_{U}\right\}_{n \in \mathbb{N}}$ is bounded in $\mathcal{F}$ and has a weakly convergent sequence in $\mathcal{F}$. Since $f_{\varepsilon} \cdot \mathbf{1}_{U}$ converges to $f \cdot \mathbf{1}_{U}$ pointwise as $\varepsilon \downarrow 0$, we conclude that $f \cdot \mathbf{1}_{U} \in \mathcal{F}$.

Lemma 3.8. For $m \in \mathbb{Z}_{+}, \lambda \in \Lambda_{m}$, and $f \in \mathcal{F}$, we have $\mu_{\left\langle H_{m} f\right\rangle}\left(U_{\lambda}\right) \leq \mu_{\langle f\rangle}\left(U_{\lambda}\right)$.

Proof. Let $g=f \cdot \mathbf{1}_{U_{\lambda}}+\left(H_{m} f\right) \cdot \mathbf{1}_{K \backslash U_{\lambda}}$. Since $g=H_{m} f+\left(f-H_{m} f\right) \cdot \mathbf{1}_{U_{\lambda}}$, it follows that $g=f$ on $V_{m}$ and $g \in \mathcal{F}$ by Lemma 3.7. By combining the inequality $\mu_{\left\langle H_{m} f\right\rangle}(K) \leq \mu_{\langle g\rangle}(K)$ and Lemma 3.1, the claim holds.

Lemma 3.9. Let $m \in \mathbb{Z}_{+}$and $\lambda \in \Lambda_{m}$. Then there exists a set $\left\{b_{p q}\right\}_{p, q \in \partial U_{\lambda}}$ of real numbers such that $b_{p q}=b_{q p} \geq 0$ for all $p \neq q$ and for every $f \in \mathcal{F}$,

$$
\mu_{\left\langle H_{m} f\right\rangle}\left(U_{\lambda}\right)=\frac{1}{2} \sum_{p, q \in \partial U_{\lambda}} b_{p q}(f(p)-f(q))^{2} .
$$

Proof. From Lemmas 3.7 and $3.1, H_{m} f=0$ on $U_{\lambda}$ if $f=0$ on $\partial U_{\lambda}$. Therefore, if $f, f^{\prime} \in \mathcal{F}$ are such that $f=f^{\prime}$ on $\partial U_{\lambda}$, then $\mu_{\left\langle H_{m} f\right\rangle}\left(U_{\lambda}\right)=\mu_{\left\langle H_{m} f^{\prime}\right\rangle}\left(U_{\lambda}\right)$. Thus, for $\varphi, \psi \in l\left(\partial U_{\lambda}\right)$

$$
\mathcal{Q}(\varphi, \psi):=\mu_{\left\langle H_{m} f, H_{m} g\right\rangle}\left(U_{\lambda}\right),
$$

where $f, g \in \mathcal{F}$ satisfy $\left.f\right|_{\partial U_{\lambda}}=\varphi$ and $\left.g\right|_{\partial U_{\lambda}}=\psi$, is well-defined. From the proof of [13, Proposition 2.1.3], the claim of the lemma follows if we prove that $\mathcal{Q}$ is a Dirichlet form on the $L^{2}$ space on $\partial U_{\lambda}$ with respect to the counting measure. The bilinearity and the nonnegativity of $\mathcal{Q}$ are evident. We prove the Markov property. Let $\varphi \in l\left(\partial U_{\lambda}\right)$ and take $f \in \mathcal{F}$ such that $\left.f\right|_{\partial U_{\lambda}}=\varphi$. We define $\hat{f}=$ $(0 \vee f) \wedge 1, \widehat{H_{m} f}=\left(0 \vee H_{m} f\right) \wedge 1$, and $h=\widehat{H_{m} f} \cdot \mathbf{1}_{U_{\lambda}}+\left(H_{m} \hat{f}\right) \cdot \mathbf{1}_{K \backslash U_{\lambda}}$. Since $h=H_{m} \hat{f}+\left(\widehat{H_{m} f}-H_{m} \hat{f}\right) \cdot \mathbf{1}_{U_{\lambda}}, h$ belongs to $\mathcal{F}$ from Lemma 3.7. Moreover, since $h=\hat{f}$ on $V_{m}$, we have

$$
0 \leq \mathcal{E}(h)-\mathcal{E}\left(H_{m} \hat{f}\right)=\frac{1}{2} \mu_{\left\langle\widehat{H_{m} f}\right\rangle}\left(U_{\lambda}\right)-\frac{1}{2} \mu_{\left\langle H_{m} \hat{f}\right\rangle}\left(U_{\lambda}\right)
$$

from Lemma 3.1. Therefore, $\mu_{\left\langle H_{m} \hat{f}\right\rangle}\left(U_{\lambda}\right) \leq \mu_{\left\langle\widehat{H_{m} f}\right\rangle}\left(U_{\lambda}\right) \leq \mu_{\left\langle H_{m} f\right\rangle}\left(U_{\lambda}\right)$. This proves the Markov property of $\mathcal{Q}$. 
Let $m \in \mathbb{Z}_{+}$and $x, y \in V_{m}$. We write $x \overleftrightarrow{m} y$ if there exists $\lambda \in \Lambda_{m}$ such that $x, y \in \partial U_{\lambda}$. We say that $\gamma_{m}=\left\{x_{0}, x_{1}, \ldots, x_{M}\right\}$ with $x_{i} \in V_{m}$ is an $m$-walk connecting $x$ and $y$ if $x_{0}=x, x_{M}=y$, and $x_{i} \overleftrightarrow{m} x_{i+1}$ for every $i=0,1, \ldots, M-1$. The length $\ell_{\boldsymbol{h}}^{(m)}\left(\gamma_{m}\right)$ of $\gamma_{m}$ based on $\boldsymbol{h}$ is defined as

$$
\ell_{\boldsymbol{h}}^{(m)}\left(\gamma_{m}\right)=\sum_{i=1}^{M}\left|\boldsymbol{h}\left(x_{i}\right)-\boldsymbol{h}\left(x_{i-1}\right)\right|_{\mathbb{R}^{N}} .
$$

For $n \geq m$ and a continuous curve $\gamma \in C([0,1] \rightarrow K)$ connecting $x$ and $y$, we define an $n$-walk $\pi_{n}(\gamma)=\left\{x_{0}, x_{1}, \ldots, x_{M}\right\}$ by $x_{0}=x$ and $x_{i}=\gamma\left(t_{i}\right)$ with $t_{i}=$ $\inf \left\{t>t_{i-1} \mid \gamma(t) \in V_{n} \backslash\left\{x_{i-1}\right\}\right\}$, inductively. Here, we set $t_{0}=0$ by convention. It is evident that $\ell_{\boldsymbol{h}}^{(n)}\left(\pi_{n}(\gamma)\right)$ is nondecreasing in $n$. From Lemma 3.5,

$$
\ell_{\boldsymbol{h}}(\gamma)=\lim _{n \rightarrow \infty} \ell_{\boldsymbol{h}}^{(n)}\left(\pi_{n}(\gamma)\right) .
$$

For $n \geq m$, we define

$$
\hat{\rho}_{\boldsymbol{h}}^{(n)}(x, y)=\inf \left\{\ell_{\boldsymbol{h}}^{(n)}\left(\gamma_{n}\right) \mid \gamma_{n} \text { is an } n \text {-walk connecting } x \text { and } y\right\}
$$

and

$$
\hat{\rho}_{\boldsymbol{h}}(x, y)=\lim _{n \rightarrow \infty} \hat{\rho}_{\boldsymbol{h}}^{(n)}(x, y) .
$$

We remark that $\hat{\rho}_{\boldsymbol{h}}(x, y)=\sup _{n \geq m} \hat{\rho}_{\boldsymbol{h}}^{(n)}(x, y)$ since $\hat{\rho}_{\boldsymbol{h}}^{(n)}(x, y)$ is nondecreasing in $n$.

Proposition 3.10. For $x, y \in V_{*}, \rho_{\boldsymbol{h}}(x, y)=\hat{\rho}_{\boldsymbol{h}}(x, y)$. In other words,

$$
\inf _{\gamma} \sup _{n} \ell_{h}^{(n)}\left(\pi_{n}(\gamma)\right)=\sup _{n} \inf _{\gamma_{n}} \ell_{h}^{(n)}\left(\gamma_{n}\right),
$$

where $\gamma$ runs over all the continuous curves connecting $x$ and $y$, and $\gamma_{n}$ runs over all the $n$-walks connecting $x$ and $y$.

Proof. From the definition, the right-hand side of (3.2) is dominated by the lefthand side. We prove the converse inequality.

For $n \geq k \geq m$ and an $n$-walk $\gamma_{n}=\left\{x_{0}, x_{1}, \ldots, x_{M}\right\}$ with $x_{0}, x_{M} \in V_{m}$, let $\pi_{n, k}\left(\gamma_{n}\right)$ denote the $k$-walk $\left\{x_{0}^{\prime}, x_{1}^{\prime}, \ldots, x_{M^{\prime}}^{\prime}\right\}$ defined as $x_{0}^{\prime}=x_{0}$ and $x_{i}^{\prime}=x_{j(i)}$ with $j(i)=\inf \left\{j>j(i-1) \mid x_{j} \in V_{k} \backslash\left\{x_{i-1}^{\prime}\right\}\right\}$ for $i=1,2, \ldots$, inductively, where we set $j(0)=0$.

Let $x, y \in V_{m}$ for $m \in \mathbb{Z}_{+}$. For each $n \geq m$, there exists a self-avoiding $n$-walk $\hat{\gamma}_{n}$ that attains $\inf _{\gamma_{n}} \ell_{\boldsymbol{h}}^{(n)}\left(\gamma_{n}\right)$ on the right-hand side of (3.2), since there are only a finite number of self-avoiding $n$-walks. For any divergent increasing sequence $\{n(k)\}$ and $n \geq m$, we can take a subsequence $\left\{n\left(k_{j}\right)\right\}$ such that $n\left(k_{1}\right) \geq n$ 
and $\left\{\pi_{n\left(k_{j}\right), n}\left(\hat{\gamma}_{n\left(k_{j}\right)}\right)\right\}_{j=1}^{\infty}$ are all the same. By a diagonal argument, we can take a divergent sequence $\{n(k)\}$ such that for every $k$ and $j$ with $k \geq j \geq m$, $\pi_{n(k), j}\left(\hat{\gamma}_{n(k)}\right)=\pi_{n(j), j}\left(\hat{\gamma}_{n(j)}\right)$. Since $\left\{\pi_{n(j), j}\left(\hat{\gamma}_{n(j)}\right)\right\}_{j=m}^{\infty}$ is consistent in the sense that $\pi_{k, j}\left(\pi_{n(k), k}\left(\hat{\gamma}_{n(k)}\right)\right)=\pi_{n(j), j}\left(\hat{\gamma}_{n(j)}\right)$ for $k \geq j \geq m$, in view of Lemma 3.4 we can construct $\gamma \in C([0,1] \rightarrow K)$ and a sequence of partitions $\left\{\Delta^{(j)}: 0=t_{0}^{(j)}<\right.$ $\left.t_{1}^{(j)}<\cdots<t_{N(j)}^{(j)}=1\right\}_{j=m}^{\infty}$ such that $\Delta^{(m)} \subset \Delta^{(m+1)} \subset \cdots, \lim _{j \rightarrow \infty}\left|\Delta^{(j)}\right|=0$, and $\pi_{n(j), j}\left(\hat{\gamma}_{n(j)}\right)=\left\{\gamma\left(t_{0}^{(j)}\right), \gamma\left(t_{1}^{(j)}\right), \ldots, \gamma\left(t_{N(j)}^{(j)}\right)\right\}$ for all $j \geq m$. Then

$$
\begin{aligned}
\sup _{j} \ell_{\boldsymbol{h}}^{(j)}\left(\pi_{j}(\gamma)\right) & =\ell_{\boldsymbol{h}}(\gamma)=\sup _{j} \ell_{\boldsymbol{h}}^{(j)}\left(\pi_{n(j), j}\left(\hat{\gamma}_{n(j)}\right)\right) \leq \sup _{j} \ell_{\boldsymbol{h}}^{(n(j))}\left(\hat{\gamma}_{n(j)}\right) \\
& =\sup _{j} \inf _{\gamma_{n(j)}} \ell_{\boldsymbol{h}}^{(n(j))}\left(\gamma_{n(j)}\right)=\operatorname{supinf}_{n} \ell_{\gamma_{n}}^{(n)}\left(\gamma_{n}\right),
\end{aligned}
$$

and (3.2) holds with $=$ replaced by $\leq$.

Proposition 3.11. Let $x, y \in K$. There exists a shortest path $\gamma$ connecting $x$ and $y$.

Proof. First, we note that $\gamma$ in the proof of Proposition 3.10 is a shortest path connecting $x$ and $y$. Therefore, the claim is true for $x, y \in V_{*}$.

We prove the claim for $x, y \in K$ with $x \neq y$. For $m \in \mathbb{Z}_{+}$, we define $U_{m}(x)=$ $\bigcup_{\lambda \in \Lambda_{m}, x \in \overline{U_{\lambda}}} \overline{U_{\lambda}}$, and $U_{m}(y)$ in the same manner. We note that $\partial U_{m}(x) \subset V_{m}$. There exists $m \in \mathbb{Z}_{+}$such that $U_{n}(x) \cap U_{n}(y)=\emptyset$ for all $n \geq m$. For $n \geq m$, take $\left(x^{(n)}, y^{(n)}\right) \in \partial U_{n}(x) \times \partial U_{n}(y)$ such that $\rho_{\boldsymbol{h}}\left(x^{(n)}, y^{(n)}\right)=\min \left\{\rho_{\boldsymbol{h}}\left(x^{\prime}, y^{\prime}\right)\right.$ $\left.\left(x^{\prime}, y^{\prime}\right) \in \partial U_{n}(x) \times \partial U_{n}(y)\right\}$. Since any continuous curve $\gamma$ connecting $x$ and $y$ passes through some points of $\partial U_{n}(x)$ and $\partial U_{n}(y)$, respectively, $\rho_{\boldsymbol{h}}\left(x^{(n)}, y^{(n)}\right)$ is nondecreasing in $n$ and $\ell_{\boldsymbol{h}}(\gamma) \geq \rho_{\boldsymbol{h}}\left(x^{(n)}, y^{(n)}\right)$. Therefore,

$$
\rho_{\boldsymbol{h}}(x, y) \geq \rho_{\boldsymbol{h}}\left(x^{(n)}, y^{(n)}\right) \quad \text { for } n \geq m \text {. }
$$

If $\rho_{\boldsymbol{h}}\left(x^{(n)}, y^{(n)}\right)=\infty$ for some $n$, the claim is trivially true. We assume that $\rho_{\boldsymbol{h}}\left(x^{(n)}, y^{(n)}\right)<\infty$ for every $n$. For $n \geq m$, take a shortest path $\gamma_{n} \in C([0,1] \rightarrow K)$ connecting $x^{(n)}$ and $y^{(n)}$. For each $n$ and $k$ with $n \geq k \geq m$, we define $s_{n, k}=$ $\inf \left\{t \in[0,1] \mid \gamma_{n}(t) \in V_{k}\right\}, t_{n, k}=\sup \left\{t \in[0,1] \mid \gamma_{n}(t) \in V_{k}\right\}, x^{(n, k)}=\gamma_{n}\left(s_{n, k}\right)$, and $y^{(n, k)}=\gamma_{n}\left(t_{n, k}\right)$. Since $V_{k}$ is a finite set, by a diagonal argument, we can take $\left\{x_{k}\right\}_{k=m}^{\infty},\left\{y_{k}\right\}_{k=m}^{\infty} \subset K$ and an increasing sequence $\{n(l)\}_{l=0}^{\infty}$ of natural numbers such that $n(0) \geq m, x_{k}, y_{k} \in V_{k}$ for all $k$, and $x^{(n(l), k)}=x_{k}$ and $y^{(n(l), k)}=y_{k}$ for all $l$ and $k$ with $l \geq k-m \geq 0$. Define $\gamma \in C([0,1] \rightarrow K)$ by connecting and reparametrizing $\left.\gamma_{n(l)}\right|_{\left[s_{n(l), l+m}, s_{n(l), l+m-1}\right]}(l=\ldots, 3,2,1),\left.\gamma_{n(0)}\right|_{\left[s_{n(0), m}, t_{n(0), m}\right]}$, and $\left.\gamma_{n(l)}\right|_{\left[t_{n(l), l+m-1}, t_{n(l), l+m}\right]}(l=1,2,3, \ldots)$. Then $\gamma$ connects $x$ and $y$ and passes through all $x_{n}$ and $y_{n}(n \geq m)$. By construction, $\ell_{\boldsymbol{h}}\left(\left.\gamma\right|_{\left[s_{n}, t_{n}\right]}\right)=\rho_{\boldsymbol{h}}\left(x_{n}, y_{n}\right)$, where 
$s_{n}$ and $t_{n}$ are the times such that $\gamma\left(s_{n}\right)=x_{n}$ and $\gamma\left(t_{n}\right)=y_{n}$. Thus, we have

$$
\ell_{\boldsymbol{h}}(\gamma)=\lim _{n \rightarrow \infty} \ell_{\boldsymbol{h}}\left(\left.\gamma\right|_{\left[s_{n}, t_{n}\right]}\right)=\lim _{n \rightarrow \infty} \rho_{\boldsymbol{h}}\left(x_{n}, y_{n}\right)
$$

Combining this with (3.3), we obtain $\ell_{\boldsymbol{h}}(\gamma) \leq \rho_{\boldsymbol{h}}(x, y)$. Therefore, $\gamma$ is a shortest path connecting $x$ and $y$.

We remark that identity (3.4) is true even if $\rho_{\boldsymbol{h}}\left(x^{(n)}, y^{(n)}\right)=\infty$ for some $n$.

Corollary 3.12. Let $x$ and $y$ be distinct elements of $K$. Then there exist sequences $\left\{x_{n}\right\}_{n=m}^{\infty}$ and $\left\{y_{n}\right\}_{n=m}^{\infty}$ in $K$ for some $m$ such that

$$
x_{n}, y_{n} \in V_{n} \quad \text { for all } n, \quad \lim _{n \rightarrow \infty} d_{K}\left(x_{n}, x\right)=\lim _{n \rightarrow \infty} d_{K}\left(y_{n}, y\right)=0
$$

and

$$
\rho_{\boldsymbol{h}}(x, y)=\lim _{k \rightarrow \infty}\left(\lim _{n \rightarrow \infty} \rho_{\boldsymbol{h}}\left(x_{k}, y_{n}\right)\right) .
$$

Proof. Take a shortest path $\gamma$ connecting $x$ and $y,\left\{x_{n}\right\},\left\{y_{n}\right\} \subset K$, and $\left\{s_{n}\right\},\left\{t_{n}\right\}$ $\subset[0,1]$ as in the proof of Proposition 3.11. Then (3.5) holds and

$$
\rho_{\boldsymbol{h}}(x, y)=\ell_{\boldsymbol{h}}(\gamma)=\lim _{k \rightarrow \infty}\left(\lim _{n \rightarrow \infty} \ell_{\boldsymbol{h}}\left(\left.\gamma\right|_{\left[s_{k}, t_{n}\right]}\right)\right)=\lim _{k \rightarrow \infty}\left(\lim _{n \rightarrow \infty} \rho_{\boldsymbol{h}}\left(x_{k}, y_{n}\right)\right) .
$$

Lemma 3.13. For each $x \in K, \mathrm{~d}_{\boldsymbol{h}}(x, y) \in[0,+\infty]$ is continuous in $y \in K$.

Proof. Let $x \in K$ and $M>0$. There exists a maximal element of

$$
\mathcal{D}=\left\{f \in \mathcal{F} \mid f(x)=0, f \leq M, \text { and } \mu_{\langle f\rangle} \leq \mu_{\langle\boldsymbol{h}\rangle}\right\},
$$

in the sense that there exists $g \in \mathcal{D}$ such that $g \geq f \mu$-a.e. for all $f \in \mathcal{D}$. Indeed, from Lemma 3.1, it suffices to take $f_{1}, f_{2}, \ldots$ from $\mathcal{D}$ such that $\int_{K} f_{k} d \mu$ converges increasingly to $\sup \left\{\int_{K} f d \mu \mid f \in \mathcal{D}\right\}$ and define $g$ as $\sup _{k} f_{k}$. Since $\mathcal{F} \subset C(K)$, $g \geq f$ on $K$ for all $f \in \mathcal{D}$. By the definition of $\mathrm{d}_{\boldsymbol{h}}, g$ is identical to $\mathrm{d}_{\boldsymbol{h}}(x, \cdot) \wedge M$. This proves the claim.

Proof of Theorem 2.2. We divide the proof into two steps.

Step 1: $x, y \in V_{m}$ for some $m \in \mathbb{Z}_{+}$. Take $n \geq m$. We define $\varphi_{n}(z):=\hat{\rho}_{\boldsymbol{h}}^{(n)}(x, z)$ for $z \in V_{n}$. Then

$$
\left|\varphi_{n}(z)-\varphi_{n}\left(z^{\prime}\right)\right| \leq\left|\boldsymbol{h}(z)-\boldsymbol{h}\left(z^{\prime}\right)\right|_{\mathbb{R}^{N}} \quad \text { for } z, z^{\prime} \in V_{n} \text { with } z \overleftrightarrow{n} z^{\prime}
$$

Indeed, there exists an $n$-walk $\gamma_{n}=\left\{x_{0}, x_{1}, \ldots, x_{M}\right\}$ connecting $x$ and $z$ such that $\varphi_{n}(z)=\ell_{\boldsymbol{h}}^{(n)}\left(\gamma_{n}\right)$. Since $\gamma_{n}^{\prime}:=\left\{x_{0}, x_{1}, \ldots, x_{M}, z^{\prime}\right\}$ is an $n$-walk connecting $x$ and $z^{\prime}$, we have

$$
\varphi_{n}\left(z^{\prime}\right) \leq \ell_{\boldsymbol{h}}^{(n)}\left(\gamma_{n}^{\prime}\right)=\varphi_{n}(z)+\left|\boldsymbol{h}(z)-\boldsymbol{h}\left(z^{\prime}\right)\right|_{\mathbb{R}^{N}} .
$$

By exchanging the roles of $z$ and $z^{\prime}$, we obtain (3.6). 
Let $\lambda \in \Lambda_{n}$ and take $\left\{b_{p q}\right\}_{p, q \in \partial U_{\lambda}}$ in Lemma 3.9. We denote $H_{n} \varphi_{n}$ by $f_{n}$. Then

$$
\begin{aligned}
\mu_{\left\langle f_{n}\right\rangle}\left(U_{\lambda}\right) & =\frac{1}{2} \sum_{p, q \in \partial U_{\lambda}} b_{p q}\left(f_{n}(p)-f_{n}(q)\right)^{2} \\
& \leq \frac{1}{2} \sum_{p, q \in \partial U_{\lambda}} b_{p q}|\boldsymbol{h}(p)-\boldsymbol{h}(q)|_{\mathbb{R}^{N}}^{2} \quad(\text { from }(3.6)) \\
& =\frac{1}{2} \sum_{j=1}^{N} \sum_{p, q \in \partial U_{\lambda}} b_{p q}\left(h_{j}(p)-h_{j}(q)\right)^{2}=\sum_{j=1}^{N} \mu_{\left\langle H_{n} h_{j}\right\rangle}\left(U_{\lambda}\right) \\
& \leq \mu_{\langle\boldsymbol{h}\rangle}\left(U_{\lambda}\right) \quad(\text { from Lemma 3.8). }
\end{aligned}
$$

In particular, $\mu_{\left\langle f_{n}\right\rangle}\left(U_{\lambda}\right) \leq \mu_{\langle\boldsymbol{h}\rangle}\left(U_{\lambda}\right)$ for all $\lambda \in \bigcup_{l=0}^{n} \Lambda_{l}$. Since $\sup _{n} \mathcal{E}\left(f_{n}\right) \leq$ $\mu_{\langle\boldsymbol{h}\rangle}(K) / 2$, the sequence $\left\{f_{n} \wedge M\right\}_{n=m}^{\infty}$ is bounded in $\mathcal{F}$ for any $M>0$. There exists a subsequence $\left\{f_{n(k)} \wedge M\right\}_{k=1}^{\infty}$ whose Cesàro means converge strongly in $\mathcal{F}$. Denoting the limit by $f^{M}$, we have

$$
\begin{aligned}
\mu_{\left\langle f^{M}\right\rangle}\left(U_{\lambda}\right)^{1 / 2} & =\lim _{k \rightarrow \infty} \mu_{\left\langle\frac{1}{k} \sum_{j=1}^{k}\left(f_{n(j)} \wedge M\right)\right\rangle}\left(U_{\lambda}\right)^{1 / 2} \\
& \leq \liminf _{k \rightarrow \infty} \frac{1}{k} \sum_{j=1}^{k} \mu_{\left\langle f_{n(j)}\right\rangle}\left(U_{\lambda}\right)^{1 / 2} \leq \mu_{\langle\boldsymbol{h}\rangle}\left(U_{\lambda}\right)^{1 / 2}
\end{aligned}
$$

for all $\lambda \in \bigcup_{l=0}^{\infty} \Lambda_{l}$. Therefore, $\mu_{\left\langle f^{M}\right\rangle} \leq \mu_{\langle\boldsymbol{h}\rangle}$ by the monotone class theorem. Since convergence in $\mathcal{F}$ yields uniform convergence from $(\mathrm{A} 2), f^{M}(x)=0$ and $f^{M}(y)=\rho_{\boldsymbol{h}}(x, y) \wedge M$ from Proposition 3.10. Thus,

$$
\mathrm{d}_{\boldsymbol{h}}(x, y) \geq f^{M}(y)-f^{M}(x)=\rho_{\boldsymbol{h}}(x, y) \wedge M .
$$

Since $M$ is arbitrary, we obtain $\mathrm{d}_{\boldsymbol{h}}(x, y) \geq \rho_{\boldsymbol{h}}(x, y)$.

Step 2: $x, y \in K$. We may assume that $x \neq y$. Take $\left\{x_{n}\right\},\left\{y_{n}\right\} \subset K$ as in Corollary 3.12. Then, from Lemma 3.13, Step 1, and Corollary 3.12,

$$
\mathrm{d}_{\boldsymbol{h}}(x, y)=\lim _{k \rightarrow \infty}\left(\lim _{n \rightarrow \infty} \mathrm{d}_{\boldsymbol{h}}\left(x_{k}, y_{n}\right)\right) \geq \lim _{k \rightarrow \infty}\left(\lim _{n \rightarrow \infty} \rho_{\boldsymbol{h}}\left(x_{k}, y_{n}\right)\right)=\rho_{\boldsymbol{h}}(x, y) .
$$

The following is a remark on the topologies of $K$ induced by $\rho_{\boldsymbol{h}}$ and $\mathrm{d}_{\boldsymbol{h}}$.

Corollary 3.14. Suppose that $\rho_{\boldsymbol{h}}$ is a $[0,+\infty]$-valued metric on $K$. Then $\mathrm{d}_{\boldsymbol{h}}$ is also a $[0,+\infty]$-valued metric. Moreover, both $\rho_{\boldsymbol{h}}$ and $\mathrm{d}_{\boldsymbol{h}}$ provide the same topology on $K$ as the original one.

Proof. From Theorem 2.2, the first claim follows and the topology $\mathcal{O}_{\boldsymbol{h}}$ associated with $\mathrm{d}_{\boldsymbol{h}}$ is stronger than that with $\rho_{\boldsymbol{h}}$. From Lemma $3.13, \mathcal{O}_{\boldsymbol{h}}$ is weaker than the 
original topology on $K$. Since a continuous bijective map from a compact Hausdorff space to a Hausdorff space is homeomorphic, by applying this fact to the identity map from $\left(K, d_{K}\right)$ to $\left(K, \rho_{\boldsymbol{h}}\right)$, the second assertion holds.

\section{§4. Proof of Theorem 2.3}

Throughout this section, we assume (B1)-(B4). Furthermore, we follow the notation used in Section 2.

For $w \in W_{m}$ with $m \in \mathbb{Z}_{+}, V_{w}$ denotes $K_{w} \cap V_{m}$. For $w=w_{1} \cdots w_{m} \in W_{m}$ and $w^{\prime}=w_{1}^{\prime} \cdots w_{m^{\prime}}^{\prime} \in W_{m^{\prime}}, w_{1} \cdots w_{m} w_{1}^{\prime} \cdots w_{m^{\prime}}^{\prime} \in W_{m+m^{\prime}}$ is represented as $w w^{\prime}$. For $i \in S, i^{n} \in W_{n}$ and $i^{\infty} \in \Sigma$ denote $\underbrace{i \cdots i}_{n}$ and $i i i \cdots$, respectively.

The Dirichlet forms associated with regular harmonic structures have a property stronger than (A2): there exists $c>0$ such that

$$
\left(\sup _{y \in K} f(y)-\inf _{x \in K} f(x)\right)^{2} \leq c \mathcal{E}(f), \quad f \in \mathcal{F} .
$$

In particular, by using Theorem 2.2, $\rho_{\boldsymbol{h}}(x, y) \leq \mathrm{d}_{\boldsymbol{h}}(x, y) \leq \sqrt{c \mu_{\langle\boldsymbol{h}\rangle}(K) / 2}<\infty$ for any $x, y \in K$.

Let $p \in V_{0}$ and take $i \in S_{0}$ such that $\psi_{i}(p)=p$. Recall that $v_{i}$ is an eigenvector of $A_{i}$ whose components are all nonnegative. Let $u_{i}$ be the column vector $\left(D_{p^{\prime} p}\right)_{p^{\prime} \in V_{0}}$. Then $u_{i}$ is an eigenvector of ${ }^{t} A_{i}$ with respect to the eigenvalue $r_{i}$ ([10, Lemma 5]). Since $K \backslash V_{0}$ is connected by (B2), (B3), and [13, Proposition 1.6.8], from [13, Theorem 3.2.11] we have

$$
u_{i}(q)>0 \quad \text { for all } q \in V_{0} \backslash\{p\} .
$$

We normalize $v_{i}$ so that $\left(u_{i}, v_{i}\right)_{l\left(V_{0}\right)}=1$. The element of $l\left(V_{0}\right)$ taking constant value 1 will be denoted by $\mathbf{1}$. Let $\tilde{l}\left(V_{0}\right)=\left\{u \in l\left(V_{0}\right) \mid(u, \mathbf{1})_{l\left(V_{0}\right)}=0\right\}$ and let $P: l\left(V_{0}\right) \rightarrow l\left(V_{0}\right)$ be the orthogonal projection onto $\tilde{l}\left(V_{0}\right)$. We note that $u_{i} \in \tilde{l}\left(V_{0}\right)$ by $D \mathbf{1}=0$ and the definition of $u_{i}$.

Lemma 4.1 (cf. $[10$, Lemma 6$])$. Let $u \in l\left(V_{0}\right)$. Then

$$
\lim _{n \rightarrow \infty} r_{i}^{-n} P A_{i}^{n} u=\left(u_{i}, u\right)_{l\left(V_{0}\right)} P v_{i} .
$$

In particular, for $q_{1}, q_{2} \in V_{0}$,

$$
\lim _{n \rightarrow \infty} r_{i}^{-n}\left(A_{i}^{n} u\left(q_{1}\right)-A_{i}^{n} u\left(q_{2}\right)\right)=\left(u_{i}, u\right)_{l\left(V_{0}\right)}\left(v_{i}\left(q_{1}\right)-v_{i}\left(q_{2}\right)\right) .
$$

Both convergences are uniform on the set $\left\{\left.u \in l\left(V_{0}\right)|| P u\right|_{l\left(V_{0}\right)} \leq 1\right\}$.

We recall a property of energy measures: 
Lemma 4.2 (cf. [6, Lemma 3.11]). For $f \in \mathcal{F}$ and $m \in \mathbb{Z}_{+}$, we have

$$
\mu_{\langle f\rangle}=\sum_{w \in W_{m}} \frac{1}{r_{w}}\left(\psi_{w}\right)_{*} \mu_{\left\langle\psi_{w}^{*} f\right\rangle},
$$

that is, $\mu_{\langle f\rangle}(A)=\sum_{w \in W_{m}} \frac{1}{r_{w}} \mu_{\left\langle\psi_{w}^{*}\right\rangle}\left(\psi_{w}^{-1}(A)\right)$ for any Borel subset $A$ of $K$.

The following is a rough upper estimate of $d_{\boldsymbol{h}}$ by $\rho_{\boldsymbol{h}}$.

Lemma 4.3. Let $m \in \mathbb{Z}_{+}, w \in W_{m}$, and $x, y \in V_{w}$ with $x \neq y$. Let $\gamma \in$ $C([0,1] \rightarrow K)$ be a shortest path connecting $x$ and $y$, and suppose that the image of $\gamma$ is contained in $K_{w}$. For each $n \in \mathbb{N}$, define $z_{n} \in V_{m+n}$ by

$$
z_{n}=\gamma\left(s_{n}\right) \quad \text { with } \quad s_{n}=\inf \left\{t \in(0,1] \mid \gamma(t) \in V_{m+n} \backslash\{x\}\right\} .
$$

Then for each $n \in \mathbb{N}$ there exists $c_{0}(n)>0$, independent of $m, w, x, y, \gamma$, such that $\rho_{\boldsymbol{h}}\left(x, z_{n}\right) \geq c_{0}(n) \mathrm{d}_{\boldsymbol{h}}(x, y)$.

Proof. Let $p \in V_{0}$ and take $i \in S_{0}$ such that $\psi_{i}(p)=p$. Let $q$ and $q^{\prime}$ denote the distinct elements of $V_{0} \backslash\{p\}$, that is, $V_{0}=\left\{p, q, q^{\prime}\right\}$. Define $\alpha \in \tilde{l}\left(V_{0}\right)$ by $\alpha(p)=1, \alpha(q)=-1$, and $\alpha\left(q^{\prime}\right)=0$. From (4.2), $\alpha$ and $u_{i}$ are linearly independent in $l\left(V_{0}\right)$; thus, the linear span of $\alpha$ and $u_{i}$ is $\tilde{l}\left(V_{0}\right)$ since $\operatorname{dim} \tilde{l}\left(V_{0}\right)=2$ from (B1). Therefore, there exists $\delta>0$ such that any $u \in l\left(V_{0}\right)$ with $|P u|_{l\left(V_{0}\right)}=1$ satisfies $\left|(u, \alpha)_{l\left(V_{0}\right)}\right| \geq \delta$ or $\left|\left(u, u_{i}\right)_{l\left(V_{0}\right)}\right| \geq \delta$. Let $\hat{q}$ denote $q$ or $q^{\prime}$. From Lemma 4.1,

$$
A_{i}^{n} u(\hat{q})-A_{i}^{n} u(p)=r_{i}^{n}\left(u_{i}, u\right)_{l\left(V_{0}\right)} v_{i}(\hat{q})+o\left(r_{i}^{n}\right) \quad \text { as } n \rightarrow \infty
$$

uniformly on $\left\{\left.u \in l\left(V_{0}\right)|| P u\right|_{l\left(V_{0}\right)}=1\right\}$. Therefore, for sufficiently large $M \in \mathbb{N}$,

$$
\left|A_{i}^{M} u(\hat{q})-A_{i}^{M} u(p)\right|_{l\left(V_{0}\right)} \geq \frac{r_{i}^{M} \delta}{2} v_{i}(\hat{q}) \quad(>0)
$$

for any $u \in l\left(V_{0}\right)$ such that $|P u|_{l\left(V_{0}\right)}=1$ and $\left|\left(u, u_{i}\right)_{l\left(V_{0}\right)}\right| \geq \delta$.

From this argument, the map

$$
\tilde{l}\left(V_{0}\right) \ni u \mapsto\left((u, \alpha)_{l\left(V_{0}\right)}^{2}+\left|A_{i}^{M} u(\hat{q})-A_{i}^{M} u(p)\right|_{l\left(V_{0}\right)}^{2}\right)^{1 / 2} \in \mathbb{R}
$$

defines a norm on $\tilde{l}\left(V_{0}\right)$; so do the maps $u \mapsto \mathcal{E}(\iota(u))^{1 / 2}$ and $u \mapsto \mathcal{E}\left(\psi_{i^{n}}^{*}(\iota(u))\right)^{1 / 2}$ for $n \in \mathbb{N}$ because of (B4). Hence, there exist $c_{1}>0$ and $c_{2}>0$ such that for every $h \in \mathcal{H}$,

$$
\mathcal{E}(h) \leq c_{1} \mathcal{E}\left(\psi_{i^{n}}^{*}(h)\right)
$$

and

$$
\mathcal{E}(h) \leq c_{2}\left((h(q)-h(p))^{2}+\left(\left(\psi_{i^{M}}^{*} h\right)(\hat{q})-\left(\psi_{i^{M}}^{*} h\right)(p)\right)^{2}\right)
$$


for all $q, \hat{q} \in V_{0} \backslash\{p\}$. Since there are only finitely many choices of $p, q$, and $\hat{q}$, we can take $c_{1}$ and $c_{2}$ to be constants independent of $p, q$, and $\hat{q}$. (Note that $c_{1}$ depends on $n$.)

Now, in the setting of the claim, let $f \in \mathcal{F}$ satisfy $\mu_{\langle f\rangle} \leq \mu_{\langle\boldsymbol{h}\rangle}$. From Lemma $4.2, \mu_{\left\langle\psi_{w}^{*} f\right\rangle} \leq \mu_{\left\langle\psi_{w}^{*} \boldsymbol{h}\right\rangle}$. In particular, $\mathcal{E}\left(\psi_{w}^{*} f\right) \leq \sum_{j=1}^{N} \mathcal{E}\left(\psi_{w}^{*} h_{j}\right)$. Let $p=$ $\psi_{w}^{-1}(x) \in V_{0}$ and take $i \in S_{0}$ such that $\psi_{i}(p)=p$. Take $k, l \in S_{0} \backslash\{i\}$ such that $z_{n}=\pi\left(w i^{n} k^{\infty}\right)$ and $z_{n+M}=\pi\left(w i^{n+M} l^{\infty}\right)$, and set $q=\pi\left(k^{\infty}\right), \hat{q}=\pi\left(l^{\infty}\right)$. Then

$$
\begin{aligned}
(f(y)-f(x))^{2} & =\left(\left(\psi_{w}^{*} f\right)\left(\psi_{w}^{-1}(y)\right)-\left(\psi_{w}^{*} f\right)(p)\right)^{2} \\
\leq & c \mathcal{E}\left(\psi_{w}^{*} f\right) \quad(\text { from }(4.1)) \\
\leq & c \sum_{j=1}^{N} \mathcal{E}\left(\psi_{w}^{*} h_{j}\right) \leq c c_{1} \sum_{j=1}^{N} \mathcal{E}\left(\psi_{w i^{n}}^{*} h_{j}\right) \\
\leq & c c_{1} c_{2} \sum_{j=1}^{N}\left\{\left(\left(\psi_{w i^{n}}^{*} h_{j}\right)(q)-\left(\psi_{w i^{n}}^{*} h_{j}\right)(p)\right)^{2}\right. \\
& \left.\quad+\left(\left(\psi_{i^{M}}^{*} \psi_{w i^{n}}^{*} h_{j}\right)(\hat{q})-\left(\psi_{i^{M}}^{*} \psi_{w i^{n}}^{*} h_{j}\right)(p)\right)^{2}\right\} \\
& =c c_{1} c_{2} \sum_{j=1}^{N}\left\{\left(h_{j}\left(z_{n}\right)-h_{j}(x)\right)^{2}+\left(h_{j}\left(z_{n+M}\right)-h_{j}(x)\right)^{2}\right\} \\
\leq & 2 c c_{1} c_{2} \rho_{\boldsymbol{h}}\left(x, z_{n}\right)^{2} .
\end{aligned}
$$

Thus, $\mathrm{d}_{\boldsymbol{h}}(x, y) \leq\left(2 c c_{1} c_{2}\right)^{1 / 2} \rho_{\boldsymbol{h}}\left(x, z_{n}\right)$.

Corollary 4.4. In the notation of Lemma 4.3, we have

(i) $\rho_{\boldsymbol{h}}(x, y) \geq c_{0}(n) \mathbf{d}_{\boldsymbol{h}}(x, y)$;

(ii) $\rho_{\boldsymbol{h}}\left(x, z_{n}\right) \geq c_{0}(n) \rho_{\boldsymbol{h}}(x, y)$.

Proof. From Lemma 4.3, (i) is evident since $\rho_{\boldsymbol{h}}(x, y) \geq \rho_{\boldsymbol{h}}\left(x, z_{n}\right)$; and (ii) follows from Lemma 4.3 and Theorem 2.2.

The following technical lemma is used in the proof of Lemma 4.6.

Lemma 4.5. Let $p \in V_{0}$ and $i \in S_{0}$ satisfy $\psi_{i}(p)=p$. Let $\alpha_{1}, \ldots, \alpha_{N} \in l\left(V_{0}\right)$ and $q \in V_{0} \backslash\{p\}$. For $n \in \mathbb{Z}_{+}$, let $\gamma_{j}^{(n)}=\left(A_{i}^{n} \alpha_{j}(q)-A_{i}^{n} \alpha_{j}(p)\right) / v_{i}(q)$ for $j=1, \ldots, N$ and $\gamma^{(n)}=\left(\sum_{j=1}^{N}\left(\gamma_{j}^{(n)}\right)^{2}\right)^{1 / 2}$. Write $\varphi_{j}^{(n)}=\iota\left(A_{i}^{n} \alpha_{j}\right)-\gamma_{j}^{(n)} \iota\left(v_{i}\right) \in \mathcal{H}$. Then, given $\delta>0$ and $\varepsilon>0$, there exists $n_{0} \in \mathbb{N}$, independent of $\alpha_{1}, \ldots, \alpha_{N}, p, q$, such that for all $n \geq n_{0}$,

$$
\mathcal{E}\left(\varphi_{j}^{(n)}\right) \leq \varepsilon\left(\gamma^{(n)}\right)^{2} \mathcal{E}\left(\iota\left(v_{i}\right)\right), \quad j=1, \ldots, N,
$$

as long as $\left|\left(u_{i}, \alpha_{l}\right)_{l\left(V_{0}\right)}\right| \geq \delta\left(\sum_{j=1}^{N}\left|P \alpha_{j}\right|_{l\left(V_{0}\right)}^{2}\right)^{1 / 2}$ for some $l \in\{1, \ldots, N\}$. 
Proof. By multiplying by a constant if necessary, we may assume the additional constraint $\sum_{j=1}^{N}\left|P \alpha_{j}\right|_{l\left(V_{0}\right)}^{2}=1$ to prove (4.3) without loss of generality. From Lemma 4.1 , for $j=1, \ldots, N$,

$$
\lim _{n \rightarrow \infty} r_{i}^{-n} P A_{i}^{n} \alpha_{j}=\left(u_{i}, \alpha_{j}\right)_{l\left(V_{0}\right)} P v_{i}
$$

and

$$
\lim _{n \rightarrow \infty} r_{i}^{-n} \gamma_{j}^{(n)}=\left(u_{i}, \alpha_{j}\right)_{l\left(V_{0}\right)}
$$

uniformly on $\Gamma:=\left\{\left.\left(\alpha_{1}, \ldots, \alpha_{N}\right) \in\left(l\left(V_{0}\right)\right)^{N}\left|\sum_{j=1}^{N}\right| P \alpha_{j}\right|_{l\left(V_{0}\right)} ^{2}=1\right\}$. Therefore,

$$
\lim _{n \rightarrow \infty} r_{i}^{-2 n} \mathcal{E}\left(\varphi_{j}^{(n)}\right)=0 \quad \text { uniformly on } \Gamma \text {. }
$$

By the assumption $\left|\left(u_{i}, \alpha_{l}\right)_{l\left(V_{0}\right)}\right| \geq \delta$ and (4.5),

$$
r_{i}^{-2 n}\left(\gamma^{(n)}\right)^{2} \geq r_{i}^{-2 n}\left(\gamma_{l}^{(n)}\right)^{2} \geq \delta^{2} / 2 \quad \text { for sufficiently large } n \text {. }
$$

Therefore, the assertion follows from (4.6), (4.7), and $\inf _{i \in S_{0}} \mathcal{E}\left(\iota\left(v_{i}\right)\right)>0$.

The following is a key lemma for the proof of Theorem 2.3.

Lemma 4.6. Let $m \in \mathbb{Z}_{+}, w \in W_{m}$, and $x \in V_{w}$. Take $i \in S_{0}$ such that $x=$ $\pi\left(w i^{\infty}\right)$. Let $\delta, \varepsilon>0$. Suppose that there exists $l \in\{1, \ldots, N\}$ such that

$$
\left|\left(u_{i}, \alpha_{w, l}\right)_{l\left(V_{0}\right)}\right| \geq \delta\left(\sum_{j=1}^{N}\left|P \alpha_{w, j}\right|_{l\left(V_{0}\right)}^{2}\right)^{1 / 2}, \quad \text { where } \quad \alpha_{w, j}=\iota^{-1}\left(\psi_{w}^{*} h_{j}\right) .
$$

Then there exists $M \in \mathbb{N}$, independent of $m, w, x, l$, such that for $n \geq M$,

$$
\mathrm{d}_{\boldsymbol{h}}(x, y) \leq(1+\varepsilon)|\boldsymbol{h}(y)-\boldsymbol{h}(x)|_{\mathbb{R}^{N}} \leq(1+\varepsilon) \rho_{\boldsymbol{h}}(x, y)
$$

for any $y \in V_{w i^{n}} \backslash\{x\}$.

Proof. For $s \in S_{0}$, let $p_{s}$ denote the fixed point of $\psi_{s}$, that is, $p_{s}=\pi\left(s^{\infty}\right)$. Let

$$
C=\max _{s \in S_{0}}\left\{\frac{\max _{q \in V_{0} \backslash\left\{p_{s}\right\}} v_{s}(q) \times\left(-D v_{s}\right)(q)}{\min _{q \in V_{0} \backslash\left\{p_{s}\right\}} v_{s}(q) \times\left(-D v_{s}\right)(q)}\right\},
$$

which is positive by (B3). Take $\varepsilon_{1}, \varepsilon_{2}>0$ such that

$$
(1+C)\left(1+\varepsilon_{2}\right)^{1 / 2}-C \leq 1+\varepsilon \quad \text { and } \quad \varepsilon_{1}=\varepsilon_{2} / 2 .
$$

We remark that any $y \in V_{w i^{n}} \backslash\{x\}$ for $n \in \mathbb{Z}_{+}$can be written as $y=\pi\left(w i^{n} k^{\infty}\right)$ for some $k \in S_{0} \backslash\{i\}$. 
Fix $k \in S_{0} \backslash\{i\}$. For $n \in \mathbb{N}$ and $j=1, \ldots, N$, let $\hat{h}_{j}^{(n)}$ denote $\psi_{w i^{n}}^{*} h_{j}$. Note that $\hat{h}_{j}^{(n)}$ can also be written as $\iota\left(A_{i}^{n} \alpha_{w, j}\right)$. Let

$$
g_{j}^{(n)}=\left(\left(\hat{h}_{j}^{(n)}\left(p_{k}\right)-\hat{h}_{j}^{(n)}\left(p_{i}\right)\right) / v_{i}\left(p_{k}\right)\right) \iota\left(v_{i}\right) \quad \text { and } \quad \varphi_{j}^{(n)}=\hat{h}_{j}^{(n)}-g_{j}^{(n)}
$$

for $j=1, \ldots, N$, and

$$
g^{(n)}=\left(\sum_{j=1}^{N}\left(\frac{\hat{h}_{j}^{(n)}\left(p_{k}\right)-\hat{h}_{j}^{(n)}\left(p_{i}\right)}{v_{i}\left(p_{k}\right)}\right)^{2}\right)^{1 / 2} \iota\left(v_{i}\right) .
$$

We note that

$$
\sum_{j=1}^{N} \mu_{\left\langle g_{j}^{(n)}\right\rangle}=\mu_{\left\langle g^{(n)}\right\rangle}
$$

and

$$
\mu_{\left\langle\hat{h}_{j}^{(n)}\right\rangle} \leq\left(1+\varepsilon_{1}\right) \mu_{\left\langle g_{j}^{(n)}\right\rangle}+\left(1+\varepsilon_{1}^{-1}\right) \mu_{\left\langle\varphi_{j}^{(n)}\right\rangle}, \quad j=1, \ldots, N .
$$

From Lemma 4.5 with $\varepsilon=\varepsilon_{1} /\left(\left(1+\varepsilon_{1}^{-1}\right) N\right)$, there exists $M \in \mathbb{N}$, independent of $m, w, x, l, k$, such that for all $n \geq M$,

$$
\mathcal{E}\left(\varphi_{j}^{(n)}\right) \leq \frac{\varepsilon_{1}}{\left(1+\varepsilon_{1}^{-1}\right) N} \mathcal{E}\left(g^{(n)}\right), \quad j=1, \ldots, N .
$$

Hereafter, we fix such an $n$ and omit the superscript ${ }^{(n)}$ from the notation. From (4.9) and (4.11), we have

$$
\sum_{j=1}^{N}\left\{\left(1+\varepsilon_{1}\right) \mu_{\left\langle g_{j}\right\rangle}(K)+\left(1+\varepsilon_{1}^{-1}\right) \mu_{\left\langle\varphi_{j}\right\rangle}(K)\right\} \leq\left(1+\varepsilon_{2}\right) \mu_{\langle g\rangle}(K) .
$$

Let $f \in \mathcal{F}$ satisfy $\mu_{\langle f\rangle} \leq \mu_{\langle\boldsymbol{h}\rangle}$ and $f(x)=0$. Let $\hat{f}$ denote $\psi_{w i^{n}}^{*} f$ and define $\check{f}:=\hat{f} \vee g \in \mathcal{F}$. Then

$$
\mu_{\langle\hat{f}\rangle} \leq \sum_{j=1}^{N} \mu_{\left\langle\hat{h}_{j}\right\rangle} \leq \sum_{j=1}^{N}\left\{\left(1+\varepsilon_{1}\right) \mu_{\left\langle g_{j}\right\rangle}+\left(1+\varepsilon_{1}^{-1}\right) \mu_{\left\langle\varphi_{j}\right\rangle}\right\}
$$

and

$$
\frac{d \mu_{\langle\check{f}\rangle}}{d \nu} \leq \frac{d \mu_{\langle\hat{f}\rangle}}{d \nu} \vee \frac{d \mu_{\langle g\rangle}}{d \nu} \quad \nu \text {-a.e. with } \nu=\mu_{\langle\hat{f}\rangle}+\mu_{\langle g\rangle}
$$

in view of Lemma 4.2, (4.10), and Lemma 3.1(ii). Combining these inequalities with (4.9), we have

$$
\mu_{\langle\breve{f}\rangle} \leq \sum_{j=1}^{N}\left\{\left(1+\varepsilon_{1}\right) \mu_{\left\langle g_{j}\right\rangle}+\left(1+\varepsilon_{1}^{-1}\right) \mu_{\left\langle\varphi_{j}\right\rangle}\right\} .
$$

In particular, $\mu_{\langle\check{f}\rangle}(K) \leq\left(1+\varepsilon_{2}\right) \mu_{\langle g\rangle}(K)$ from (4.12). 
Let $F=\left(1+\varepsilon_{2}\right)^{-1 / 2} \iota\left(\left.\check{f}\right|_{V_{0}}\right)\left(=\left(1+\varepsilon_{2}\right)^{-1 / 2} H_{0} \check{f}\right) \in \mathcal{H}$. Then we have $\mathcal{E}(F) \leq$ $\mathcal{E}\left(\left(1+\varepsilon_{2}\right)^{-1 / 2} \check{f}\right) \leq \mathcal{E}(g)$, which implies that

$$
0 \leq \mathcal{E}(F-g)=\mathcal{E}(F)-2 \mathcal{E}(F, g)+\mathcal{E}(g) \leq 2 \mathcal{E}(g-F, g) .
$$

Letting $G=g-F \in \mathcal{H}$, we have $G\left(p_{i}\right)=0$ and

$$
G(q)=g(q)-\left(1+\varepsilon_{2}\right)^{-1 / 2}(\hat{f}(q) \vee g(q)) \leq\left(1-\left(1+\varepsilon_{2}\right)^{-1 / 2}\right) g(q)
$$

for any $q \in V_{0}$. Let $q^{\prime}$ denote the unique element of $V_{0} \backslash\left\{p_{i}, p_{k}\right\}$. Since

$$
\left(\left.G\right|_{V_{0}},-D v_{i}\right)_{l\left(V_{0}\right)}=\left(\sum_{j=1}^{N}\left(\frac{\hat{h}_{j}\left(p_{k}\right)-\hat{h}_{j}\left(p_{i}\right)}{v_{i}\left(p_{k}\right)}\right)^{2}\right)^{-1 / 2} \mathcal{E}(g-F, g) \geq 0
$$

we have

$$
\begin{aligned}
G\left(p_{k}\right)\left(-D v_{i}\right)\left(p_{k}\right) & \geq-G\left(p_{i}\right)\left(-D v_{i}\right)\left(p_{i}\right)-G\left(q^{\prime}\right)\left(-D v_{i}\right)\left(q^{\prime}\right) \\
& \geq 0-\left(1-\left(1+\varepsilon_{2}\right)^{-1 / 2}\right) g\left(q^{\prime}\right)\left(-D v_{i}\right)\left(q^{\prime}\right) \\
& \geq-C\left(1-\left(1+\varepsilon_{2}\right)^{-1 / 2}\right) g\left(p_{k}\right)\left(-D v_{i}\right)\left(p_{k}\right) .
\end{aligned}
$$

Thus,

$$
\begin{aligned}
-C\left(1-\left(1+\varepsilon_{2}\right)^{-1 / 2}\right) g\left(p_{k}\right) & \leq G\left(p_{k}\right)=g\left(p_{k}\right)-\left(1+\varepsilon_{2}\right)^{-1 / 2}\left(\hat{f}\left(p_{k}\right) \vee g\left(p_{k}\right)\right) \\
& \leq g\left(p_{k}\right)-\left(1+\varepsilon_{2}\right)^{-1 / 2} \hat{f}\left(p_{k}\right),
\end{aligned}
$$

which implies that

$$
\hat{f}\left(p_{k}\right) \leq\left((1+C)\left(1+\varepsilon_{2}\right)^{1 / 2}-C\right) g\left(p_{k}\right) \leq(1+\varepsilon) g\left(p_{k}\right) .
$$

Therefore, for $y=\pi\left(w i^{n} k^{\infty}\right) \in V_{w i^{n}} \backslash\{x\}$,

$$
\begin{aligned}
f(y)-f(x) & =f(y) \leq(1+\varepsilon)\left(\sum_{j=1}^{N}\left(\frac{\hat{h}_{j}\left(p_{k}\right)-\hat{h}_{j}\left(p_{i}\right)}{v_{i}\left(p_{k}\right)}\right)^{2}\right)^{1 / 2} v_{i}\left(p_{k}\right) \\
& =(1+\varepsilon)|\boldsymbol{h}(y)-\boldsymbol{h}(x)|_{\mathbb{R}^{N}} \leq(1+\varepsilon) \rho_{\boldsymbol{h}}(x, y) .
\end{aligned}
$$

By taking the supremum with respect to $f$, we obtain (4.8).

Lemma 4.7. There exists $\delta^{\prime}>0$ such that for any distinct points $i, j$ of $S_{0}$ and every $u \in l\left(V_{0}\right),\left|\left(u_{i}, u\right)_{l\left(V_{0}\right)}\right| \vee\left|\left(u_{j}, u\right)_{l\left(V_{0}\right)}\right| \geq \delta^{\prime}|P u|_{l\left(V_{0}\right)}$.

Proof. Since the linear span of $u_{i}$ and $u_{j}$ is $\tilde{l}\left(V_{0}\right)$,

$$
\inf \left\{\left.\left|\left(u_{i}, u\right)_{l\left(V_{0}\right)}\right| \vee\left|\left(u_{j}, u\right)_{l\left(V_{0}\right)}\right|\left|u \in \tilde{l}\left(V_{0}\right),\right| u\right|_{l\left(V_{0}\right)}=1\right\}>0 .
$$

Therefore, the assertion follows. 
Now, we prove Theorem 2.3. For the proof, we make a slight generalization of the concept of $\ell_{\boldsymbol{h}}$. Let $\mathcal{I}$ be a disjoint union of a finite set $\left\{I_{k}\right\}$ of closed intervals. For $\gamma \in C(\mathcal{I} \rightarrow K)$, we define its length $\ell_{\boldsymbol{h}}(\gamma)$ by $\sum_{k} \ell_{\boldsymbol{h}}\left(\left.\gamma\right|_{I_{k}}\right)$.

Proof of Theorem 2.3. From Theorem 2.2, it suffices to prove that $\rho_{\boldsymbol{h}}(x, y) \geq$ $\mathrm{d}_{\boldsymbol{h}}(x, y)$ for distinct $x, y \in K$.

Step 1: $x, y \in V_{m}$ for some $m \in \mathbb{Z}_{+}$. Take $\delta^{\prime}$ as in Lemma 4.7. Let $\varepsilon>0, \delta=\delta^{\prime} / \sqrt{N}$ and take $M$ as in Lemma 4.6. Take a shortest path $\gamma \in C([0,1] \rightarrow K)$ connecting $x$ and $y$. We may assume that $\gamma$ is injective. Let $\mathcal{I}_{1}=[0,1]$. We define $\left\{I_{n, k}\right\}_{k=1}^{l(n)}$, $\left\{J_{n, k}\right\}_{k=1}^{l(n)}$, and $\mathcal{I}_{n+1}$ for $n \in \mathbb{N}$ inductively as follows. First, let $\left\{I_{n, k}\right\}_{k=1}^{l(n)}$ be the collection of closed intervals $I_{n, k}=\left[s_{n, k}, t_{n, k}\right]$ such that:

- $\bigcup_{k=1}^{l(n)} I_{n, k}=\mathcal{I}_{n}$.

- $s_{n, k}<t_{n, k}, \gamma\left(s_{n, k}\right) \in V_{m+M n}, \gamma\left(t_{n, k}\right) \in V_{m+M n}$, and $\gamma(t) \notin V_{m+M n}$ for all $t \in\left(s_{n, k}, t_{n, k}\right)$.

- For $k \neq k^{\prime}, I_{n, k} \cap I_{n, k^{\prime}}$ consists of at most one point.

Next, for $k=1, \ldots, l(n)$, take $w \in W_{m+M n}$ and $i, \hat{i} \in S_{0}$ such that $\gamma\left(\left[s_{n, k}, t_{n, k}\right]\right)$ $\subset K_{w}, \gamma\left(s_{n, k}\right)=\pi\left(w i^{\infty}\right)$, and $\gamma\left(t_{n, k}\right)=\pi\left(w \hat{i}^{\infty}\right)$. Denote $\iota^{-1}\left(\psi_{w}^{*} h_{j}\right)$ by $\alpha_{j}$ for $j=1, \ldots, N$. Take $j \in\{1, \ldots, N\}$ such that $\left|P \alpha_{j}\right|_{l\left(V_{0}\right)}$ attains the maximum of $\left\{\left|P \alpha_{1}\right|_{l\left(V_{0}\right)}, \ldots,\left|P \alpha_{N}\right|_{l\left(V_{0}\right)}\right\}$. From Lemma 4.7, at least one of the following holds:

(i) $\left|\left(u_{i}, \alpha_{j}\right)_{l\left(V_{0}\right)}\right| \geq \delta^{\prime}\left|P \alpha_{j}\right|_{l\left(V_{0}\right)}$;

(ii) $\left|\left(u_{\hat{i}}, \alpha_{j}\right)_{l\left(V_{0}\right)}\right| \geq \delta^{\prime}\left|P \alpha_{j}\right|_{l\left(V_{0}\right)}$.

If (i) holds, set $J_{n, k}=\left[s_{n, k}, t_{n, k}^{\prime}\right]$ with $t_{n, k}^{\prime}=\inf \left\{t>s_{n, k} \mid \gamma(t) \in V_{m+M(n+1)}\right\}$. Otherwise, set $J_{n, k}=\left[s_{n, k}^{\prime}, t_{n, k}\right]$ with $s_{n, k}^{\prime}=\sup \left\{t<t_{n, k} \mid \gamma(t) \in V_{m+M(n+1)}\right\}$. Define $\mathcal{I}_{n+1}=\bigcup_{k=1}^{l(n)} \overline{I_{n, k} \backslash J_{n, k}}$.

Let $n \in \mathbb{N}$ and $k=1, \ldots, l(n)$. From Corollary 4.4(ii),

$$
\ell_{\boldsymbol{h}}\left(\left.\gamma\right|_{J_{n, k}}\right) \geq c_{0}(M) \ell_{\boldsymbol{h}}\left(\left.\gamma\right|_{I_{n, k}}\right)
$$

that is,

Therefore,

$$
\ell_{\boldsymbol{h}}\left(\left.\gamma\right|_{I_{n, k} \backslash J_{n, k}}\right) \leq\left(1-c_{0}(M)\right) \ell_{\boldsymbol{h}}\left(\left.\gamma\right|_{I_{n, k}}\right)
$$

$$
\ell_{\boldsymbol{h}}\left(\left.\gamma\right|_{\mathcal{I}_{n+1}}\right) \leq\left(1-c_{0}(M)\right) \ell_{\boldsymbol{h}}\left(\left.\gamma\right|_{\mathcal{I}_{n}}\right)
$$

Then

$$
\ell_{\boldsymbol{h}}\left(\left.\gamma\right|_{\mathcal{I}_{n}}\right) \leq\left(1-c_{0}(M)\right)^{n-1} \ell_{\boldsymbol{h}}(\gamma)=\left(1-c_{0}(M)\right)^{n-1} \rho_{\boldsymbol{h}}(x, y)
$$

Fix $R \in \mathbb{N}$ and let $\mathscr{J}=\left\{J_{n, k} \mid 1 \leq n \leq R, 1 \leq k \leq l(n)\right\}$. Let $0=t_{0}<t_{1}<$ $\cdots<t_{l}=1$ be the arrangement of all the endpoints of the intervals $J_{n, k}$ in $\mathscr{J}$ in 
increasing order. For all $i=0, \ldots, l-1$, we obtain

$$
\rho_{\boldsymbol{h}}\left(\gamma\left(t_{i}\right), \gamma\left(t_{i+1}\right)\right) \geq c_{0}(M) \mathrm{d}_{\boldsymbol{h}}\left(\gamma\left(t_{i}\right), \gamma\left(t_{i+1}\right)\right)
$$

by applying Corollary 4.4(i) to a series of adjacent points of a suitable $n$-walk connecting $\gamma\left(t_{i}\right)$ and $\gamma\left(t_{i+1}\right)$, where $n$ is the smallest number such that $\gamma\left(t_{i}\right) \in V_{n}$ and $\gamma\left(t_{i+1}\right) \in V_{n}$. Let $\mathcal{Q}=\left\{i=0, \ldots, l-1 \mid\left[t_{i}, t_{i+1}\right] \in \mathscr{J}\right\}$. From Lemma 4.6, for $i \in \mathcal{Q}$,

$$
\rho_{\boldsymbol{h}}\left(\gamma\left(t_{i}\right), \gamma\left(t_{i+1}\right)\right) \geq(1+\varepsilon)^{-1} \mathbf{d}_{\boldsymbol{h}}\left(\gamma\left(t_{i}\right), \gamma\left(t_{i+1}\right)\right) .
$$

Then

$$
\begin{aligned}
\rho_{\boldsymbol{h}}(x, y)= & \sum_{i=0}^{l-1} \rho_{\boldsymbol{h}}\left(\gamma\left(t_{i}\right), \gamma\left(t_{i+1}\right)\right) \geq \sum_{i \in \mathcal{Q}} \rho_{\boldsymbol{h}}\left(\gamma\left(t_{i}\right), \gamma\left(t_{i+1}\right)\right) \\
\geq & (1+\varepsilon)^{-1} \sum_{i \in \mathcal{Q}} \mathrm{d}_{\boldsymbol{h}}\left(\gamma\left(t_{i}\right), \gamma\left(t_{i+1}\right)\right) \\
\geq & (1+\varepsilon)^{-1} \sum_{i=0}^{l-1} \mathrm{~d}_{\boldsymbol{h}}\left(\gamma\left(t_{i}\right), \gamma\left(t_{i+1}\right)\right) \\
& -(1+\varepsilon)^{-1} c_{0}(M)^{-1} \sum_{i \notin \mathcal{Q}} \rho_{\boldsymbol{h}}\left(\gamma\left(t_{i}\right), \gamma\left(t_{i+1}\right)\right) \quad(\text { from }(4.14)) \\
\geq & (1+\varepsilon)^{-1} \mathrm{~d}_{\boldsymbol{h}}(x, y)-(1+\varepsilon)^{-1} c_{0}(M)^{-1} \ell_{\boldsymbol{h}}\left(\left.\gamma\right|_{\mathcal{I}_{R+1}}\right) \\
\geq & (1+\varepsilon)^{-1} \mathrm{~d}_{\boldsymbol{h}}(x, y)-(1+\varepsilon)^{-1} c_{0}(M)^{-1}\left(1-c_{0}(M)\right)^{R} \rho_{\boldsymbol{h}}(x, y) .
\end{aligned}
$$

Here, (4.13) was used in the last inequality. By letting $R \rightarrow \infty$ and $\varepsilon \rightarrow 0$, we conclude that $\rho_{\boldsymbol{h}}(x, y) \geq \mathrm{d}_{\boldsymbol{h}}(x, y)$.

Step 2: $x, y \in K$. Take $\left\{x_{n}\right\},\left\{y_{n}\right\} \subset K$ as in Corollary 3.12. Then, from Corollary 3.12, Step 1, and Lemma 3.13,

$$
\rho_{\boldsymbol{h}}(x, y)=\lim _{k \rightarrow \infty}\left(\lim _{n \rightarrow \infty} \rho_{\boldsymbol{h}}\left(x_{k}, y_{n}\right)\right) \geq \lim _{k \rightarrow \infty}\left(\lim _{n \rightarrow \infty} \mathrm{d}_{\boldsymbol{h}}\left(x_{k}, y_{n}\right)\right)=\mathrm{d}_{\boldsymbol{h}}(x, y) .
$$

\section{Acknowledgements}

This research was partly supported by KAKENHI $(21740094,24540170)$. The author thanks Naotaka Kajino for insightful discussions and the anonymous referee for very careful reading and valuable proposals which have led to improvements of the first version.

\section{References}

[1] Z.-Q. Chen and M. Fukushima, Symmetric Markov processes, time change, and boundary theory, London Math. Soc. Monogr. Ser. 35, Princeton Univ. Press, Princeton, NJ, 2012. Zbl 1253.60002 MR 2849840 
[2] E. B. Davies, Heat kernels and spectral theory, Cambridge Tracts in Math. 92, Cambridge Univ. Press, Cambridge, 1990. Zbl 0699.35006 MR 0990239

[3] G. De Cecco and G. Palmieri, Integral distance on a Lipschitz Riemannian manifold, Math. Z. 207 (1991), 223-243. Zbl 0722.58006 MR 1109664

[4] R. Engelking, General topology, Monografie Mat. 60, PWN-Polish Scientific Publishers, Warszawa, 1977. Zbl 0373.54002 MR 0500780

[5] M. Fukushima, Y. Oshima, and M. Takeda, Dirichlet forms and symmetric Markov processes, 2nd revised and extended ed., de Gruyter Stud. Math. 19, Gruyter, Berlin, 2011. Zbl 1227.31001 MR 2778606

[6] M. Hino, On singularity of energy measures on self-similar sets, Probab. Theory Related Fields 132 (2005), 265-290. Zbl 1097.31005 MR 2199293

[7] _ Martingale dimensions for fractals, Ann. Probab. 36 (2008), 971-991. Zbl 1144.60046 MR 2408580

[8] _ Energy measures and indices of Dirichlet forms, with applications to derivatives on some fractals, Proc. London Math. Soc. (3) 100 (2010), 269-302. Zbl 1185.60089 MR 2578475

[9] , Measurable Riemannian structures associated with strong local Dirichlet forms, Math. Nachr. 286 (2013), 1466-1478. Zbl 1277.31017 MR 3119694

[10] M. Hino and K. Nakahara, On singularity of energy measures on self-similar sets. II, Bull. London Math. Soc. 38 (2006), 1019-1032. Zbl 1109.60034 MR 2285256

[11] N. Kajino, Heat kernel asymptotics for the measurable Riemannian structure on the Sierpinski gasket, Potential Anal. 36 (2012), 67-115. Zbl 1248.28012 MR 2886454

[12] J. Kigami, Harmonic metric and Dirichlet form on the Sierpiński gasket, in Asymptotic problems in probability theory: stochastic models and diffusions on fractals (Sanda/Kyoto, 1990), Pitman Res. Notes Math. Ser. 283, Longman Sci. Tech., Harlow, 1993, 201-218. Zbl 0793.31005 MR 1354156

[13] _ Analysis on fractals, Cambridge Tracts in Math. 143, Cambridge Univ. Press, Cambridge, 2001. Zbl 0998.28004 MR 1840042

[14] _ Measurable Riemannian geometry on the Sierpinski gasket: the Kusuoka measure and the Gaussian heat kernel estimate, Math. Ann. 340 (2008), 781-804. Zbl 1143.28004 MR 2372738

[15] S. Kusuoka, Dirichlet forms on fractals and products of random matrices, Publ. RIMS Kyoto Univ. 25 (1989), 659-680. Zbl 0694.60071 MR 1025071

[16] T. Lindstrøm, Brownian motion on nested fractals, Mem. Amer. Math. Soc. 83 (1990), no. 420. Zbl 0688.60065 MR 0988082

[17] V. Metz and K.-T. Sturm, Gaussian and non-Gaussian estimates for heat kernels on the Sierpiński gasket, in: Dirichlet forms and stochastic processes (Beijing, 1993), de Gruyter, Berlin, 1995, 283-289. Zbl 0841.31008 MR 1366443

[18] J. R. Norris, Heat kernel asymptotics and the distance function in Lipschitz Riemannian manifolds, Acta Math. 179 (1997), 79-103. Zbl 0912.58041 MR 1484769

[19] K.-T. Sturm, Analysis on local Dirichlet spaces. II. Upper Gaussian estimates for the fundamental solutions of parabolic equations, Osaka J. Math. 32 (1995), 275-312. Zbl 0854.35015 MR 1355744

[20] A. Teplyaev, Gradients on fractals, J. Funct. Anal. 174 (2000), 128-154. Zbl 0956.31008 MR 1761365

[21] Harmonic coordinates on fractals with finitely ramified cell structure, Canad. J. Math. 60 (2008), 457-480. Zbl 1219.28012 MR 2398758 